\title{
Development of Regional Curves Relating Bankfull-Channel Geometry and Discharge to Drainage Area for Streams in Pennsylvania and Selected Areas of Maryland
}

By Jeffrey J. Chaplin

In cooperation with the Pennsylvania Department of Environmental Protection, the Canaan Valley Institute, and the Susquehanna River Basin Commission

Scientific Investigations Report 2005-5147 


\title{
U.S. Department of the Interior Gale A. Norton, Secretary
}

\author{
U.S. Geological Survey \\ P. Patrick Leahy, Acting Director
}

\section{U.S. Geological Survey, Reston, Virginia: 2005}

For sale by U.S. Geological Survey, Information Services

Box 25286, Denver Federal Center

Denver, CO 80225

For more information about the USGS and its products:

Telephone: 1-888-ASK-USGS

World Wide Web: http://www.usgs.gov/

Any use of trade, product, or firm names in this publication is for descriptive purposes only and does not imply endorsement by the U.S. Government.

Although this report is in the public domain, permission must be secured from the individual copyright owners to reproduce any copyrighted materials contained within this report.

Chaplin, J.J., 2005, Development of regional curves relating bankfull-channel geometry and discharge to drainage area for streams in Pennsylvania and selected areas of Maryland: U.S. Geological Survey Scientific Investigations Report 2005-5147, $34 \mathrm{p}$. 


\section{Contents}

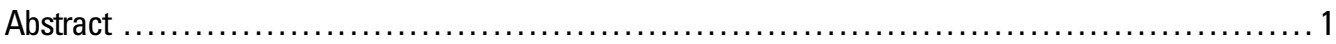

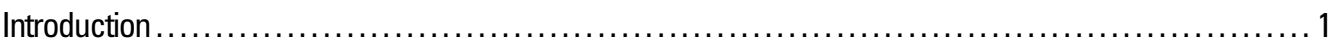

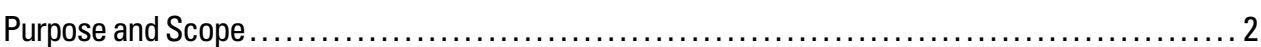

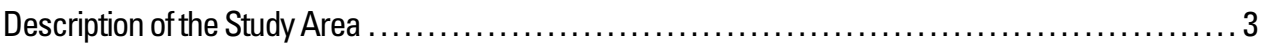

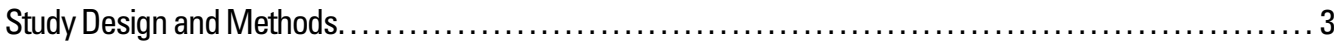

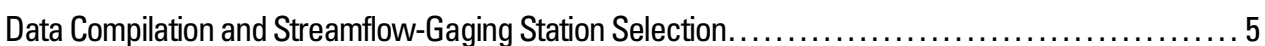

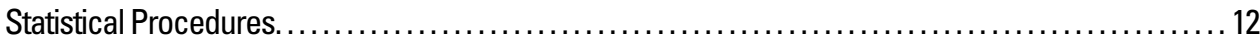

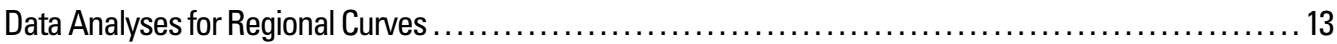

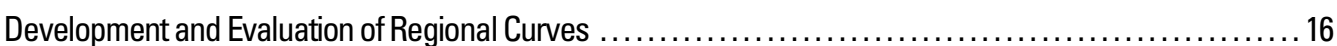

Regional Curves Developed for Noncarbonate Settings $\ldots \ldots \ldots \ldots \ldots \ldots \ldots \ldots \ldots \ldots \ldots \ldots \ldots \ldots \ldots \ldots \ldots \ldots \ldots \ldots \ldots$

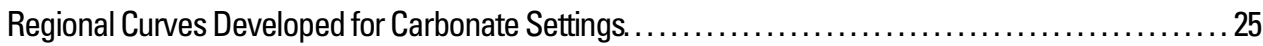

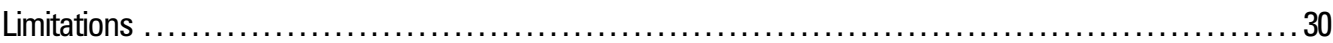

Limitations Related to Selection of Streamflow-Gaging Stations and the Bankfull Channel .......... 30

Limitations Related to Development of Regional Curves................................. 31

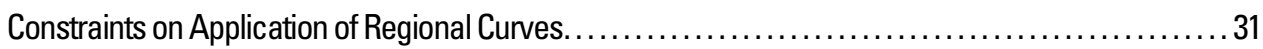

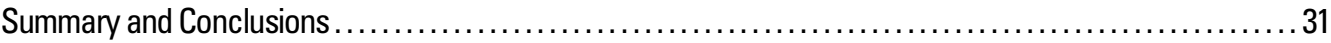

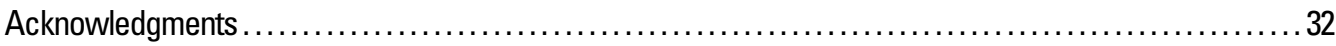

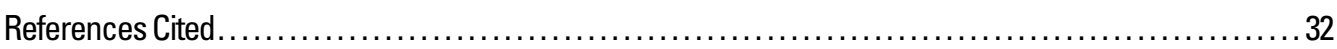

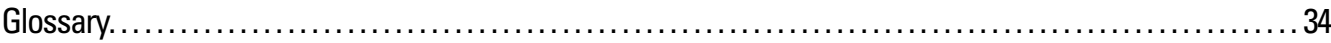

Appendix 1 Photo-documentation, bankfull-channel geometry, and substrate data collected from streams selected for development of regional curves (InPocket)

\section{Figures}

1. Map showing locations of physiographic provinces, sections, and U.S. Geological Survey streamflow-gaging stations selected for regional-curve development, Pennsylvania and Maryland...

2. Photograph showing streamflow-gaging station and hypothetical example of a bankfull-channel elevation used for development of regional curves in Pennsylvania and selected areas of Maryland.

3-12. Graphs showing:

3. Relation between partial residuals for percent of watershed area underlain by carbonate bedrock and the percent of watershed area underlain by carbonate bedrock........... 15

4. Relation between bankfull discharge and drainage area with emphasis on the influence of carbonate bedrock, Pennsylvania and selected areas of Maryland................. 17

5. Regional curve representing the relation between bankfull cross-sectional area and drainage area in noncarbonate settings of Pennsylvania and selected areas of Maryland .. 21

6. Regional curve representing the relation between bankfull discharge and drainage area in noncarbonate settings of Pennsylvania and selected areas of Maryland .............. 22

7. Regional curve representing the relation between bankfull width and drainage area in noncarbonate settings of Pennsylvania and selected areas of Maryland. 


\section{Figures-Continued}

8. Regional curve representing the relation between bankfull mean depth and drainage area in noncarbonate settings of Pennsylvania and selected areas of Maryland ....

9. Regional curve representing the relation between bankfull cross-sectional area and drainage area for carbonate settings of Pennsylvania and selected areas of Maryland .... . 26

10. Regional curve representing the relation between bankfull discharge and drainage area for carbonate settings of Pennsylvania and selected areas of Maryland ............ 27

11. Regional curve representing the relation between bankfull width and drainage area for carbonate settings of Pennsylvania and selected areas of Maryland.................. 28

12. Regional curve representing the relation between bankfull mean depth and drainage area for carbonate settings of Pennsylvania and selected areas of Maryland....

\section{Tables}

1. Physiographic characteristics and percentage of carbonate bedrock underlying watersheds of streamflow-gaging stations and associated stream reaches assessed for regional-curve development, Pennsylvania and selected areas of Maryland ....

2. Statistical results from analysis of covariance of regressions relating bankfull-channel characteristics to drainage area in watersheds with and without carbonate bedrock in the Piedmont, Ridge and Valley, and Appalachian Plateaus Physiographic Provinces.

3. Statistical results from analysis of covariance of regressions relating bankfull-channel characteristics to drainage area in watersheds without carbonate bedrock in the Piedmont, Ridge and Valley, and Appalachian Plateaus Physiographic Provinces.

4. Channel characteristics from assessment of streamflow-gaging stations and associated stream reaches in noncarbonate settings of Pennsylvania and selected areas of Maryland. ....

5. Channel characteristics from assessment of streamflow-gaging stations and associated stream reaches in carbonate settings of Pennsylvania and selected areas of Maryland.

6. Coefficients and diagnostic statistics for regional curves relating bankfull characteristics to drainage area in noncarbonate settings of Pennsylvania and selected areas of Maryland ...... 25

7. Coefficients and diagnostic statistics for regional curves relating bankfull characteristics to drainage area in carbonate settings of Pennsylvania and selected areas of Maryland 


\section{Conversion Factors and Datum}

\begin{tabular}{|c|c|c|}
\hline Multiply & By & To obtain \\
\hline \multicolumn{3}{|c|}{ Length } \\
\hline inch (in.) & 25.4 & millimeter (mm) \\
\hline foot $(\mathrm{ft})$ & 0.3048 & meter $(\mathrm{m})$ \\
\hline mile (mi) & 1.609 & kilometer $(\mathrm{km})$ \\
\hline \multicolumn{3}{|c|}{ Area } \\
\hline square foot $\left(\mathrm{ft}^{2}\right)$ & 929.0 & square centimeter $\left(\mathrm{cm}^{2}\right)$ \\
\hline square foot $\left(\mathrm{ft}^{2}\right)$ & 0.09290 & square meter $\left(\mathrm{m}^{2}\right)$ \\
\hline square mile $\left(\mathrm{mi}^{2}\right)$ & 2.590 & square kilometer $\left(\mathrm{km}^{2}\right)$ \\
\hline \multicolumn{3}{|c|}{ Flow rate } \\
\hline cubic foot per second $\left(\mathrm{ft}^{3} / \mathrm{s}\right)$ & 0.02832 & cubic meter per second $\left(\mathrm{m}^{3} / \mathrm{s}\right)$ \\
\hline
\end{tabular}

Horizontal coordinate information is referenced to the North American Datum of 1927 (NAD 27). 



\title{
Development of Regional Curves Relating Bankfull- Channel Geometry and Discharge to Drainage Area for Streams in Pennsylvania and Selected Areas of Maryland
}

\author{
By Jeffrey J. Chaplin
}

\section{Abstract}

Natural-stream designs are commonly based on the dimensions of the bankfull channel, which is capable of conveying discharges that transport sediment without excessive erosion or deposition. Regional curves relate bankfull-channel geometry and discharge to drainage area in watersheds with similar runoff characteristics and commonly are utilized by practitioners of natural-stream design to confirm or refute selection of the fieldidentified bankfull channel. Data collected from 66 streamflowgaging stations and associated stream reaches between December 1999 and December 2003 were used in one-variable ordinary least-squares regression analyses to develop regional curves relating drainage area to cross-sectional area, discharge, width, and mean depth of the bankfull channel. Watersheds draining to these stations are predominantly within the Piedmont, Ridge and Valley, and Appalachian Plateaus Physiographic Provinces of Pennsylvania and northern Maryland.

Statistical analyses of physiography, percentage of watershed area underlain by carbonate bedrock, and percentage of watershed area that is glaciated indicate that carbonate bedrock, not physiography or glaciation, has a controlling influence on the slope of regional curves. Regional curves developed from stations in watersheds underlain by 30 percent or less carbonate bedrock generally had steeper slopes than the corresponding relations developed from watersheds underlain by greater than 30 percent carbonate bedrock. In contrast, there is little evidence to suggest that regional curves developed from stations in the Piedmont or Ridge and Valley Physiographic Province are different from the corresponding relations developed from stations in the Appalachian Plateaus Physiographic Province. On the basis of these findings, regional curves are presented to represent two settings that are independent of physiography:

(1) noncarbonate settings characterized by watersheds with carbonate bedrock underlying 30 percent or less of watershed area, and (2) carbonate settings characterized by watersheds with carbonate bedrock underlying greater than 30 percent of watershed area.

All regional curves presented in this report have slopes that are significantly different from zero and normally distributed residuals that vary randomly with drainage area. Drainage area explains the most variability in bankfull cross-sectional area and bankfull discharge in the noncarbonate setting $\left(\mathrm{R}^{2}=0.92\right.$ for both). Less variability is explained in bankfull width and depth $\left(R^{2}=0.81\right.$ and 0.72 , respectively). Regional curves representing the carbonate setting are generally not as statistically robust as the corresponding noncarbonate relations because there were only 11 stations available to develop these curves and drainage area cannot explain variance resulting from karst features. The carbonate regional curves generally are characterized by less confidence, lower $\mathrm{R}^{2}$ values, and higher residual standard errors. Poor representation by watersheds less than $40 \mathrm{mi}^{2}$ causes the carbonate regional curves for bankfull discharge, cross-sectional area, and mean depth to be disproportionately influenced by the smallest watershed (values of Cook's Distance range from 3.6 to 8.4). Additional bankfull discharge and channel-geometry data from small watersheds might reduce this influence, increase confidence, and generally improve regional curves representing the carbonate setting.

Limitations associated with streamflow-gaging station selection and development of the curves result in some constraints for the application of regional curves presented in this report. These curves apply only to streams within the study area in watersheds having land use, streamflow regulation, and drainage areas that are consistent with the criteria used for station selection. Regardless of the setting, the regional curves presented here are not intended for use as the sole method for estimation of bankfull characteristics; however, they may supplement field identification of the bankfull channel when used in conjunction with field-verified bankfull indicators, streamflow-frequency analysis, or other supporting evidence.

\section{Introduction}

Restoration of stream channels that have excessive erosion, deposition, or degraded habitat is commonly proposed and implemented by federal, state, local, or private organizations in an effort to return the channels to more stable and biologically productive conditions. Traditional engineering practices for stream stabilization frequently rely on hardening reaches that are subjected to erosive forces with rip-rap, gabions, concrete, 
or other countermeasures. Stream restoration ${ }^{1}$ efforts utilizing a natural-stream design approach that emphasizes working in concert with natural stream processes as opposed to combating them have gained popularity and are now commonly practiced in many areas. These efforts are intended to restore impaired stream reaches to allow for transport of sediment load and runoff without excessive aggradation or degradation while maintaining habitat and aesthetics consistent with unimpaired reaches subjected to similar runoff conditions.

The bankfull discharge is considered to be the most effective streamflow for moving sediment, forming or changing stream-channel bars, forming or changing bends and meanders, and generally doing work that results in the average morphological characteristics of the channel (Wolman and Miller, 1960; Dunne and Leopold, 1978). The bankfull discharge is, therefore, the channel-forming discharge and is responsible for the formation of the bankfull channel. The objective of many natural-stream designs is to return sediment-transport capacity through construction of a channel that has a pattern, profile, and geometry consistent with that of the bankfull channel. Proper design relies heavily on the correct selection of the bankfull channel.

Some of the more common tools available for supporting the selection of the bankfull channel are regional curves. Regional curves are one-variable ordinary least-squares regressions relating bankfull discharge, cross-sectional area, width, and mean depth to drainage area in settings that are expected to have similar runoff characteristics. Equations describing the regional curves can be used to estimate the discharge and dimensions of the bankfull channel when drainage area of the watershed is known. These equations are useful for supporting selection of the bankfull channel in areas with similar runoff characteristics.

Physiographic provinces are often assumed to have homogenous, yet unique, runoff characteristics and are commonly used to define the area that regional curves represent. Recent studies in Maryland successfully used physiographic provinces in developing regional curves. McCandless (2003b) found that regional curves relating drainage area to bankfull discharge in the Allegheny Plateau and Ridge and Valley (McCandless, 2003a) ${ }^{2}$, Piedmont (McCandless and Everett, 2002), and Coastal Plain (McCandless, 2003b) Physiographic Provinces of Maryland were statistically different. New York State has developed regional curves for selected hydrologic regions (Westergard and others, 2005) that are delineated on the basis of physiography, geology, channel slope, basin storage, precipitation, and snow cover (Lumia, 1991). The relation between bankfull-discharge and drainage area in Westergard and others (2005, p. 14) were compared to results from other studies in the northeastern United States and southern Ontario to show that equations can vary among regions.

More localized characteristics such as the size, shape, slope, underlying geology, and distribution of landforms within a physiographic province make the runoff behavior of each watershed unique. These local characteristics are superimposed on the broader-scale physiographic setting and, in some instances, may provide a more refined means of defining settings having similar runoff characteristics. For example, streams draining watersheds underlain by carbonate bedrock commonly experience underflow and storage within karst features. As a result, Pennsylvania streams draining watersheds underlain by carbonate bedrock commonly have lower peak flows than streams in watersheds without carbonate bedrock (White, 1976; Stuckey and Reed, 2000).

The influence of physiography and geologic variables on regional curves has not been tested in the study area. Statistical analyses of data from 66 streamflow-gaging stations predominantly in the Piedmont, Ridge and Valley, and Appalachian Plateaus Physiographic Provinces of Pennsylvania and northern Maryland were used to determine which physiographic and (or) geologic variables significantly influence the slope and (or) intercept of regional curves. These analyses provide the basis for development of two sets of regional curves: (1) one set that is representative of watersheds with carbonate bedrock underlying less than or equal to $(\leq) 30$ percent of the watershed area (herein referred to as "noncarbonate"), and (2) one set that is representative of watersheds with carbonate bedrock underlying greater than (>) 30 percent of the watershed area (herein referred to as "carbonate"). The two sets of regional curves presented in this report incorporate the data from, and are intended to supersede, regional curves previously published by Cinotto (2003) and White (2001).

\section{Purpose and Scope}

This report presents the methods used, data collected, and equations describing regional curves developed for Pennsylvania and selected areas of Maryland. Bankfull discharge and channel-geometry data collected at 52 stations and associated stream reaches were combined with data from 14 stations published by Cinotto (2003) and White (2001). All data used for development of regional curves were collected between December 1999 and December 2003. Regional curves were developed by relating the following bankfull characteristics to drainage area through regression analysis: discharge, crosssectional area, width, and mean depth. An evaluation of selected physiographic and geologic variables affecting the development of regional curves also is presented. The majority of the

\footnotetext{
${ }^{1}$ Terms that are in boldface print are defined in the Glossary section of this report.
}

\footnotetext{
${ }^{2}$ The term Ridge and Valley is usage of the Pennsylvania Geological Survey (Pennsylvania Department of Conservation and Natural Resources, 1995). This terminology will also be used in the report for areas outside of Pennsylvania for consistency.
} 
watershed area draining to each station was used to assign stations to the following physiographic provinces: 12 stations are in the Piedmont Province, 2 are in the New England Province, 27 are in the Ridge and Valley Province, 24 are in the Appalachian Plateaus Province, and 1 station is in the Central Lowland Province.

Statistical analyses of relations among bankfull characteristics and selected physiographic and geologic variables were used to determine (1) if differences in the slope and (or) intercept of regression equations for the Piedmont, Ridge and Valley, and Appalachian Plateaus Physiographic Provinces justify development of separate regional curves for each province; (2) if carbonate bedrock or glaciation explain a significant amount of variance in bankfull discharge; and (3) if estimates of bankfull-channel geometry and discharge are improved by multiple-variable ordinary least-squares regression (multiple regression) using drainage area, percentage of watershed underlain by carbonate bedrock (percent carbonate), and (or) percentage of watershed area that is glaciated (percent glaciated) as explanatory variables.

\section{Description of the Study Area}

The study area includes seven physiographic provinces in Pennsylvania, and the Piedmont, Ridge and Valley, and Blue Ridge Physiographic Provinces in northern Maryland (fig. 1). The majority of gaged watersheds where data were collected are characterized as having most of the watershed area underlain by the Piedmont, Ridge and Valley, or Appalachian Plateaus Physiographic Provinces, or some combination of them. The New England, Blue Ridge, Atlantic Coastal Plain, and Central Lowland Physiographic Provinces are poorly represented and therefore are not described below.

The Piedmont Physiographic Province is made up of the Piedmont Lowland Section, the Gettysburg-Newark Lowland Section, and the Piedmont Upland Section (fig. 1). The Piedmont Lowland Section is underlain primarily by limestone and dolomite bedrock that is susceptible to formation of karst features. It is characterized by broad, moderately dissected valleys separated by broad low hills that range in altitude from 170 to $630 \mathrm{ft}$ (Sevon, 2000). Drainage is mostly dendritic, but some areas within the section have virtually no pattern because of the well-developed subsurface drainage. The Gettysburg-Newark Lowland Section consists mainly of rolling hills and valleys developed on sedimentary rocks. Isolated higher hills have developed on the diabase, sedimentary rock, and conglomerates. Almost all the underlying sedimentary rock dips to the north or northwest, and many smaller drainage ways are oriented normal to the direction of dip so that some of the topography has a northeast-southwest linearity. The basic drainage pattern is dendritic. Altitude in the section ranges from 40 to 1,335 ft. The Piedmont Upland Section consists mainly of broad, rounded to flat-topped hills and shallow valleys developed on schist, gneiss, and quartzite with some saprolite. Altitude in this section ranges from approximately $100 \mathrm{ft}$ to approx- imately 1,220 ft. The rocks are intensely folded and faulted, and the resulting drainage pattern is primarily dendritic.

The Ridge and Valley Physiographic Province is made up of the Great Valley and Appalachian Mountain Sections (fig. 1). The Great Valley Section is underlain by shale and sandstone in the northwest, slate near the eastern boundary, and limestone and dolomite throughout the southeast (Cuff and others, 1989). Underground storage and underflow is enhanced by karst features (Kochanov and Reese, 2003). Altitudes range from approximately 140 to $1,100 \mathrm{ft}$. The drainage pattern is primarily dendritic but varies because of subsurface drainage (Sevon, 2000). The Appalachian Mountain Section is underlain predominantly by sandstone, siltstone, shale, and conglomerate; however, some areas are underlain by anthracite coal or limestone and dolomite. Long parallel ridges separated by broad to linear valleys are characteristic of this section. Altitudes range from approximately 260 to $2,775 \mathrm{ft}$. Drainage patterns are varied.

The Appalachian Plateaus is the largest physiographic province in Pennsylvania and is made up of nine sections that extend over the northern and western portions of the state (fig. 1). In contrast to the linear ridges of the Ridge and Valley, the landscape is irregular and is dominated by dendritic drainage patterns (Sevon, 2000). This province is underlain primarily by sandstone, siltstone, shale, and conglomerate. However, limestone underlies parts of the Allegheny Mountain and Pittsburgh Low Plateau Sections (Sevon, 2000). Karst features are uncommon because the limestone typically is overlain by sedimentary rocks and (or) interbedded with shale (Pennsylvania Department of Conservation and Natural Resources, 2000b). The northeast and northwest sections (fig. 1) were shaped by glacial erosion and deposition, resulting in varied upland and valley features (Sevon, 2000).

\section{Study Design and Methods}

This study was designed to develop regional curves using streamflow-gaging stations in predominantly non-urban and non-regulated settings of Pennsylvania and selected areas of Maryland. Bankfull channel geometry and discharge data from 12 stations in the Piedmont Physiographic Province and 2 stations in the New England Physiographic Province previously published by White (2001) and Cinotto (2003) were combined with data collected from 52 additional stations in the Ridge and Valley, Appalachian Plateaus, and Central Lowland Physiographic Provinces. Collectively, the 66 stations represent a range of watershed areas in different physiographic and geologic settings across Pennsylvania and northern Maryland. The methods of data compilation and station selection are described below and are followed by a description of the statistical procedures used to develop the regional curves. 


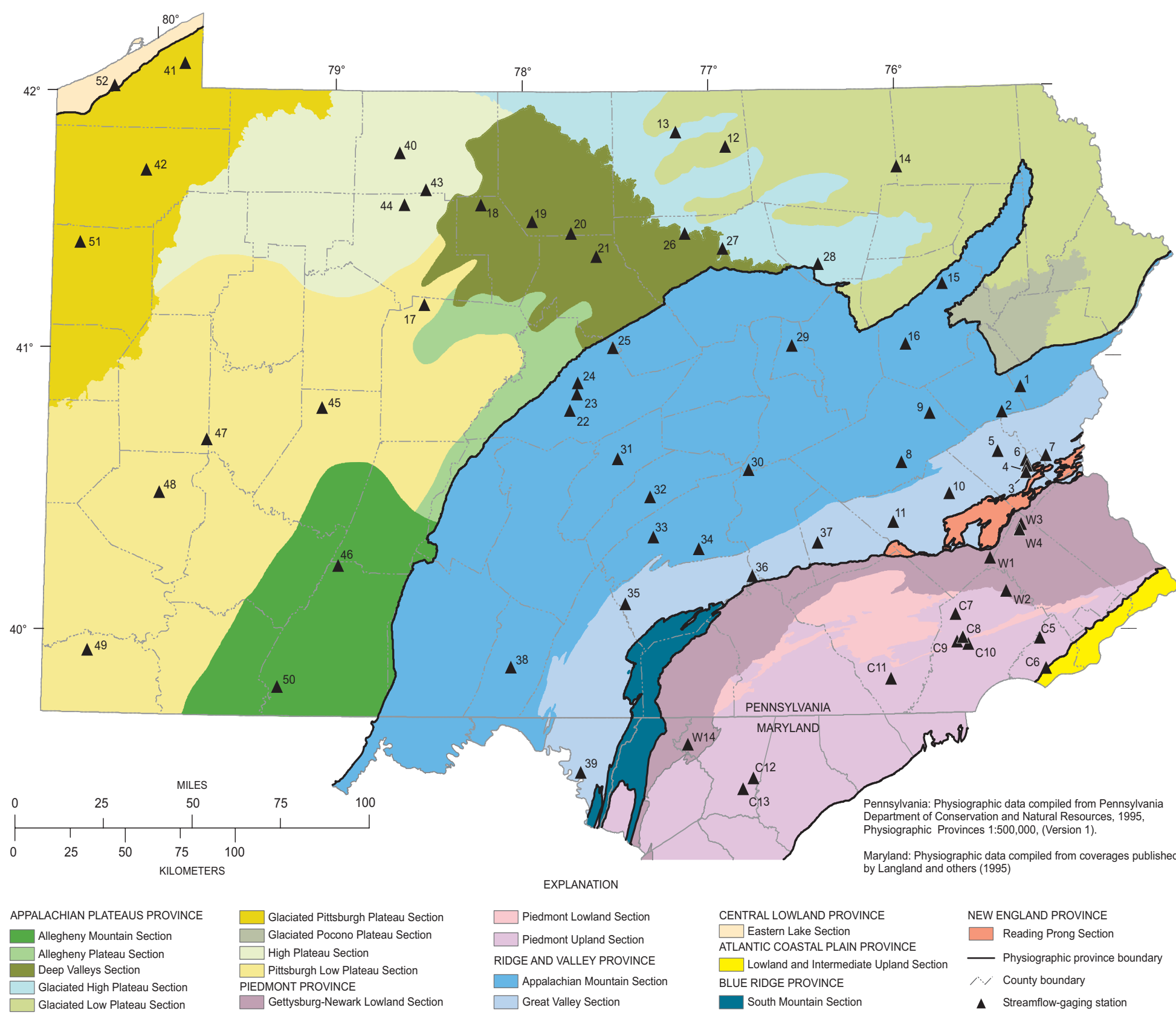

Figure 1. Locations of physiographic provinces, sections, and U.S. Geological Survey streamflow-gaging stations selected for regional-curve development, Pennsylvania and Maryland. See table 1 for information associated with cross-reference numbers identifying each station in this figure. 


\section{Data Compilation and Streamflow-Gaging Station Selection}

Bankfull-discharge and channel-geometry data were collected at streamflow-gaging stations selected mainly on the basis of seven filtering criteria. The filtering criteria are intended to provide a consistent means of selecting streamflowgaging stations that have a sufficient period of record to characterize the bankfull channel. A brief description of each criterion is provided below:

- The watershed draining to each station is predominantly within the boundaries of the Piedmont, Ridge and Valley, Appalachian Plateaus, New England, or Central Lowland Physiographic Province. Note that the predominant province is defined as the one underlaying the largest percentage of watershed area. Some portion of many watersheds is outside of predominant province (table 1).

- The watershed has not been subjected to anthracite or bituminous coal mining that alters surface and underground hydrology.

- No more than approximately 20 percent of the upstream watershed is classified as "urban" land use. This percentage was chosen for consistency with the selection criteria of White (2001), Cinotto (2003) and other efforts to develop regional curves specific to Maryland.

- The station has a period of record of at least 10 years.

- If the station is discontinued, the station must have been operational until at least 1985 to minimize the possibility of changes to the channel that would require re-development of the rating curve relating water-surface elevation to discharge (rating). Re-development of ratings is beyond the scope this study.

- Streamflow at the station is subjected to no greater than approximately 20 percent regulation. This percentage was chosen for consistency with the selection criteria of White (2001) and Cinotto (2003).

- The stream was wadeable at the time of survey. This criterion limited the study to watersheds less than $220 \mathrm{mi}^{2}$.

Stations were selected by applying these filtering criteria to a database of active and discontinued continuous- and partialrecord streamflow-gaging stations operated by the USGS in the study area. The period of record, date of discontinuance, and percent regulation of each station were determined from data stored and maintained by the USGS in the Automated Data And Processing System (ADAPS) of the National Water Information System (NWIS) (U.S. Geological Survey, 2003). A Geographic Information System (GIS) containing physiography, and land-use data was used for filtering purposes. Physiographic coverages are from the Pennsylvania Department of Conservation and Natural Resources (1995) and from Langland and others (1995). The combined physiographic coverages are shown in figure 1. Land-use data are published in Stuckey and Reed (2000) and were compiled by USGS (1996). The percentage of urban area in each watershed is defined as the sum of three land-cover classifications - low-intensity developed, high-intensity residential, and high-intensity commercial/ industrial.

Streamflow-gaging stations meeting all filtering criteria were considered for inclusion in this study. Station characteristics from records in USGS offices, communication with field personnel, and, in some instances, field reconnaissance were used to further refine the list of suitable stations by excluding those where lateral adjustment was restricted by levees or berms. Some stations that were selected had some anthropogenic influences such as rip rap, gabions, or retaining walls over short reaches. However, none were channelized or hardened with poured concrete. Bedrock outcrops occurred in, or near, the channel of some stream reaches.

Percent carbonate was used to evaluate whether or not carbonate bedrock explains a significant amount of variability in bankfull discharge. Similarly, percent glaciated was used to elevate glaciation as an explanatory variable. Percent carbonate was determined by superimposing coverages of carbonate bedrock (Pennsylvania Department of Conservation and Natural Resources, 2000b) on watershed boundaries delineated for each station. Percent glaciated was determined by superimposing coverages of the Wisconsinan glacial advance (Pennsylvania Department of Conservation and Natural Resources, 2000a) on watershed boundaries of each station.

Procedures described by Leopold (1994) and Rosgen (1996) were followed for identification of the bankfull channel, determination of bankfull discharge and channel geometry, and determination of stream type. Long-term streamflow and crosssectional channel geometry data stored in ADAPS were used in combination with bankfull field indicators identified in longitudinal and cross-sectional surveys to define the bankfull channel. Peak-flow analysis of long-term streamflow records following guidelines described by the Water Resources Council, Hydrology Committee (1981) was used to determine the exceedance probability and corresponding recurrence interval of the range of streamflows observed at each station. With the exception of station 39 (Marsh Run at Grimes, Md.), the bankfull channel is assumed to be formed by a discharge recurring once every 1 to 2 years. 
Table 1. Physiographic characteristics and percentage of carbonate bedrock underlying watersheds of streamflow-gaging stations and associated stream reaches assessed for regional-curve development, Pennsylvania and selected areas of Maryland.

[—, no carbonate bedrock; ft, feet; St., Street; D.S., downstream; U.S., upstream; RV, Ridge and Valley Province; am, Appalachian Mountain Section; gv, Great Valley Section; AP,

Appalachian Plateaus Province; alm, Allegheny Mountain Section; alp, Allegheny Plateau Section; dv, Deep Valleys Section; ghp, Glaciated High Plateau Section; glp, Glaciated Low Plateau Section; gpp, Glaciated Pittsburgh Plateau Section; gpop, Glaciated Pocono Plateau Section; hp, High Plateau Section; plp, Pittsburgh Low Plateau Section; P, Piedmont Province; gnl, Gettysburg-Newark Lowland Section; 1, Lowland Section; u, Upland Section; BR, Blue Ridge Province; sm, South Mountain Section; CL, Central Lowland Province; el, Eastern Lake Section; NE, New England Province; rp, Reading Prong Section]

\begin{tabular}{|c|c|c|c|c|c|c|}
\hline $\begin{array}{l}\text { Cross- } \\
\text { reference } \\
\text { identification } \\
\text { number }\end{array}$ & $\begin{array}{c}\text { U.S. } \\
\text { Geological } \\
\text { Survey } \\
\text { Station } \\
\text { identifi- } \\
\text { cation } \\
\text { number }\end{array}$ & Station name & $\begin{array}{l}\text { Latitude/ } \\
\text { longitude }^{1}\end{array}$ & $\begin{array}{l}\text { Physiographic unit } \\
\text { (percentage } \\
\text { underlying total } \\
\text { watershed area) }\end{array}$ & $\begin{array}{c}\text { Percentage } \\
\text { of } \\
\text { watershed } \\
\text { underlain by } \\
\text { carbonate } \\
\text { bedrock }^{3}\end{array}$ & Reach location \\
\hline 1 & 01449360 & Pohopoco Creek at Kresgeville, Pa. & $\begin{array}{l}40^{\circ} 53^{\prime} 51^{\prime \prime} \\
75^{\circ} 30^{\prime} 10^{\prime \prime}\end{array}$ & $\begin{array}{l}\text { RV - am - 89 } \\
\text { AP - gpop - } 11\end{array}$ & - & $\begin{array}{l}\text { From } 20 \mathrm{ft} \text { U.S. of station to } 1,075 \mathrm{ft} \text { D.S. of } \\
\text { station. Total length is } 1,095 \mathrm{ft} \text {. }\end{array}$ \\
\hline 2 & 01450500 & Aquashicola Creek at Palmerton, Pa. & $\begin{array}{l}40^{\circ} 48^{\prime} 22^{\prime \prime} \\
75^{\circ} 35^{\prime} 54^{\prime \prime}\end{array}$ & $\mathrm{RV}-\mathrm{am}-100$ & 5 & $\begin{array}{l}\text { From } 2,361 \mathrm{ft} \text { U.S. of station to station. Total } \\
\text { length is } 2,361 \mathrm{ft} \text {. }\end{array}$ \\
\hline 3 & 01451500 & Little Lehigh Creek near Allentown, Pa. & $\begin{array}{l}40^{\circ} 34^{\prime} 56^{\prime \prime} \\
75^{\circ} 29^{\prime} 00^{\prime \prime}\end{array}$ & $\begin{array}{l}\mathrm{RV}-\mathrm{gv}-76 \\
\mathrm{NE}-\mathrm{rp}-24\end{array}$ & 63 & $\begin{array}{l}\text { From } 2,399 \mathrm{ft} \text { U.S. of station to station. Total } \\
\text { length is } 2,399 \mathrm{ft} \text {. }\end{array}$ \\
\hline 4 & 01451650 & $\begin{array}{l}\text { Little Lehigh Creek at 10th St. Bridge at } \\
\text { Allentown, Pa. }\end{array}$ & $\begin{array}{l}40^{\circ} 35^{\prime} 47^{\prime \prime} \\
75^{\circ} 28^{\prime} 28^{\prime \prime}\end{array}$ & $\begin{array}{l}R V-g v-80 \\
N E-r p-20\end{array}$ & 70 & $\begin{array}{l}\text { From station to } 1,570 \mathrm{ft} \text { D.S. Total length is } \\
1,570 \mathrm{ft} \text {. }\end{array}$ \\
\hline 5 & 01451800 & Jordan Creek near Schnecksville, Pa. & $\begin{array}{l}40^{\circ} 39^{\prime} 42^{\prime \prime} \\
75^{\circ} 37^{\prime} 38^{\prime \prime}\end{array}$ & $\begin{array}{l}R V-g v-99 \\
R V-\text { am }-1\end{array}$ & - & $\begin{array}{l}\text { From station to } 985 \mathrm{ft} \text { D.S. of station. Total } \\
\text { length is } 985 \mathrm{ft} \text {. }\end{array}$ \\
\hline 6 & 01452000 & Jordan Creek at Allentown, $\mathrm{Pa}$. & $\begin{array}{l}40^{\circ} 37^{\prime} 23^{\prime \prime} \\
75^{\circ} 28^{\prime} 58^{\prime \prime}\end{array}$ & $R V-g v-100$ & 11 & $\begin{array}{l}\text { From } 1,860 \mathrm{ft} \text { U.S. of station to station. Total } \\
\text { length is } 1,860 \mathrm{ft} \text {. }\end{array}$ \\
\hline 7 & 01452500 & Monocacy Creek at Bethlehem, Pa. & $\begin{array}{l}40^{\circ} 38^{\prime} 28^{\prime \prime} \\
75^{\circ} 22^{\prime} 47^{\prime \prime}\end{array}$ & $R V-g v-100$ & 69 & $\begin{array}{l}\text { From station to } 1,200 \mathrm{ft} \text { D.S. of station. Total } \\
\text { length is } 1,200 \mathrm{ft} \text {. }\end{array}$ \\
\hline 8 & 01468500 & Schuylkill River at Landingville, Pa. & $\begin{array}{l}40^{\circ} 37^{\prime} 45^{\prime \prime} \\
76^{\circ} 07^{\prime} 30^{\prime \prime}\end{array}$ & RV - am - 100 & - & $\begin{array}{l}\text { From } 2,775 \mathrm{ft} \text { U.S. of station to station. Total } \\
\text { length is } 2,775 \mathrm{ft} \text {. }\end{array}$ \\
\hline 9 & 01469500 & Little Schuylkill River at Tamaqua, Pa. & $\begin{array}{l}40^{\circ} 48^{\prime} 25^{\prime \prime} \\
75^{\circ} 58^{\prime} 20^{\prime \prime}\end{array}$ & RV - am - 100 & - & $\begin{array}{l}\text { From } 722 \mathrm{ft} \text { U.S. of station to } 278 \mathrm{ft} \text { D.S. of } \\
\text { station. Total length is } 1,000 \mathrm{ft} \text {. }\end{array}$ \\
\hline 10 & 01470756 & Maiden Creek at Virginville, Pa. & $\begin{array}{l}40^{\circ} 30^{\prime} 51^{\prime \prime} \\
75^{\circ} 53^{\prime} 00^{\prime \prime}\end{array}$ & $\begin{array}{l}R V-g v-90 \\
R V-a m-5 \\
N E-r p-5\end{array}$ & 16 & $\begin{array}{l}\text { From } 156 \mathrm{ft} \mathrm{U.S.} \mathrm{of} \mathrm{station} \mathrm{to} 1,164 \mathrm{ft} \text { D.S. of } \\
\text { station. Total length is } 1,320 \mathrm{ft} \text {. }\end{array}$ \\
\hline 11 & 01470779 & Tulpehocken Creek near Bernville, Pa. & $\begin{array}{l}40^{\circ} 24^{\prime} 48^{\prime \prime} \\
76^{\circ} 10^{\prime} 19^{\prime \prime}\end{array}$ & $\begin{array}{l}\text { RV - gv - } 97 \\
\text { NE - rp - } 3\end{array}$ & 82 & $\begin{array}{l}\text { From } 30 \mathrm{ft} \text { U.S. of station to } 1,129 \mathrm{ft} \text { D.S. of } \\
\text { station. Total length is } 1,159 \mathrm{ft} \text {. }\end{array}$ \\
\hline 12 & 01516500 & Corey Creek near Mainesburg, Pa. & $\begin{array}{l}41^{\circ} 47^{\prime} 27^{\prime \prime} \\
77^{\circ} 00^{\prime} 54^{\prime \prime}\end{array}$ & $\begin{array}{l}\text { AP - glp - } 70 \\
\text { AP - ghp - } 30\end{array}$ & - & $\begin{array}{l}\text { From } 855 \mathrm{ft} \text { U.S. of station to } 37 \mathrm{ft} \mathrm{D.S} \mathrm{of} \\
\text { station. Total length is } 892 \mathrm{ft} .\end{array}$ \\
\hline 13 & 01518420 & $\begin{array}{l}\text { Crooked Creek below Catlin Hollow at } \\
\text { Middlebury Center, Pa. }\end{array}$ & $\begin{array}{l}41^{\circ} 50^{\prime} 33^{\prime \prime} \\
77^{\circ} 16^{\prime} 25^{\prime \prime}\end{array}$ & $\begin{array}{l}\text { AP - glp - } 66 \\
\text { AP - ghp - } 34\end{array}$ & - & $\begin{array}{l}\text { From } 880 \mathrm{ft} \text { U.S. of station to } 620 \mathrm{ft} \text { D.S. of } \\
\text { station. Total length is } 1,500 \mathrm{ft} .\end{array}$ \\
\hline 14 & 01533250 & Tuscarora Creek near Silvara, Pa. & $\begin{array}{l}41^{\circ} 42^{\prime} 25^{\prime \prime} \\
76^{\circ} 07^{\prime} 10^{\prime \prime}\end{array}$ & AP - glp - 100 & - & $\begin{array}{l}\text { From } 477 \mathrm{ft} \text { U.S. of station to } 473 \mathrm{ft} \text { D.S. of } \\
\text { station. Total length is } 950 \mathrm{ft} .\end{array}$ \\
\hline 15 & 01537000 & Toby Creek at Luzerne, $\mathrm{Pa}$. & $\begin{array}{l}41^{\circ} 16^{\prime} 51^{\prime \prime} \\
75^{\circ} 53^{\prime} 46^{\prime \prime}\end{array}$ & $\begin{array}{l}\text { AP - glp - } 83 \\
\text { RV - am - } 17\end{array}$ & - & $\begin{array}{l}\text { From } 600 \mathrm{ft} \text { U.S. of station to station. Total } \\
\text { length is } 600 \mathrm{ft} \text {. }\end{array}$ \\
\hline
\end{tabular}


Table 1. Physiographic characteristics and percentage of carbonate bedrock underlying watersheds of streamflow-gaging stations and associated stream reaches assessed for regional-curve development, Pennsylvania and selected areas of Maryland.-Continued

[—, no carbonate bedrock; ft, feet; St., Street; D.S., downstream; U.S., upstream; RV, Ridge and Valley Province; am, Appalachian Mountain Section; gv, Great Valley Section; AP, Appalachian Plateaus Province; alm, Allegheny Mountain Section; alp, Allegheny Plateau Section; dv, Deep Valleys Section; ghp, Glaciated High Plateau Section; glp, Glaciated Low Plateau Section; gpp, Glaciated Pittsburgh Plateau Section; gpop, Glaciated Pocono Plateau Section; hp, High Plateau Section; plp, Pittsburgh Low Plateau Section; P, Piedmont Province; gnl, Gettysburg-Newark Lowland Section; 1 , Lowland Section; u, Upland Section; BR, Blue Ridge Province; sm, South Mountain Section; CL, Central Lowland Province; el, Eastern Lake Section; NE, New England Province; rp, Reading Prong Section]

\begin{tabular}{|c|c|c|c|c|c|c|}
\hline $\begin{array}{c}\text { Cross- } \\
\text { reference } \\
\text { identification } \\
\text { number }\end{array}$ & $\begin{array}{c}\text { U.S. } \\
\text { Geological } \\
\text { Survey } \\
\text { Station } \\
\text { identifi- } \\
\text { cation } \\
\text { number }\end{array}$ & Station name & $\begin{array}{l}\text { Latitude/ } \\
\text { longitude }^{1}\end{array}$ & $\begin{array}{l}\text { Physiographic unit } \\
\text { (percentage } \\
\text { underlying total } \\
\text { watershed area) }\end{array}$ & $\begin{array}{l}\text { Percentage } \\
\text { of } \\
\text { watershed } \\
\text { underlain by } \\
\text { carbonate } \\
\text { bedrock }^{3}\end{array}$ & Reach location \\
\hline 16 & 01538000 & $\begin{array}{l}\text { Wapwallopen Creek near } \\
\text { Wapwallopen, Pa. }\end{array}$ & $\begin{array}{l}41^{\circ} 03^{\prime} 33^{\prime \prime} \\
76^{\circ} 05^{\prime} 38^{\prime \prime}\end{array}$ & $\begin{array}{l}\mathrm{RV}-\mathrm{am}-88 \\
\mathrm{AP}-\text { gpop - } 12\end{array}$ & - & $\begin{array}{l}\text { From } 940 \mathrm{ft} \text { U.S. of station to } 60 \mathrm{ft} \text { D.S. of } \\
\text { station. Total length is } 1,000 \mathrm{ft} \text {. }\end{array}$ \\
\hline 17 & 01542720 & Wilson Run at Penfield, $\mathrm{Pa}$. & $\begin{array}{l}41^{\circ} 12^{\prime} 58^{\prime \prime} \\
78^{\circ} 35^{\prime} 00^{\prime \prime}\end{array}$ & AP - plp - 100 & - & $\begin{array}{l}\text { From } 292 \mathrm{ft} \text { U.S. of station to } 344 \mathrm{ft} \text { D.S. of } \\
\text { station. Total length is } 636 \mathrm{ft} .\end{array}$ \\
\hline 18 & 01542810 & Waldy Run near Emporium, Pa. & $\begin{array}{l}41^{\circ} 34^{\prime} 44^{\prime \prime} \\
78^{\circ} 17^{\prime} 34^{\prime \prime}\end{array}$ & $A P-d v-100$ & - & $\begin{array}{l}\text { From } 747 \mathrm{ft} \text { U.S. of station to } 34 \mathrm{ft} \mathrm{D.S.} \mathrm{of} \\
\text { station. Total length is } 781 \mathrm{ft} .\end{array}$ \\
\hline 19 & 01543700 & $\begin{array}{l}\text { First Fork Sinnemahoning Creek at Whar- } \\
\text { ton, Pa. }\end{array}$ & $\begin{array}{l}41^{\circ} 31^{\prime} 08^{\prime \prime} \\
78^{\circ} 01^{\prime} 40^{\prime \prime}\end{array}$ & AP $-d v-100$ & - & $\begin{array}{l}\text { From station to } 1,615 \mathrm{ft} \text { D.S. of station Total } \\
\text { length is } 1,615 \mathrm{ft} .\end{array}$ \\
\hline 20 & 01544500 & Kettle Creek at Cross Fork, Pa. & $\begin{array}{l}41^{\circ} 28^{\prime} 33^{\prime \prime} \\
77^{\circ} 49^{\prime} 34^{\prime \prime}\end{array}$ & $A P-d v-100$ & - & $\begin{array}{l}\text { From } 72 \mathrm{ft} \mathrm{U.S.} \mathrm{of} \mathrm{station} \mathrm{to} 2,348 \mathrm{ft} \text { D.S. of } \\
\text { station. Total length is } 2,420 \mathrm{ft} \text {. }\end{array}$ \\
\hline 21 & 01545600 & Young Womans Creek near Renovo, Pa. & $\begin{array}{l}41^{\circ} 23^{\prime} 22^{\prime \prime} \\
77^{\circ} 41^{\prime} 28^{\prime \prime}\end{array}$ & AP $-d v-100$ & - & $\begin{array}{l}\text { From } 493 \mathrm{ft} \text { U.S. of station to } 707 \mathrm{ft} \text { D.S. of } \\
\text { station. Total length is } 1,200 \mathrm{ft} \text {. }\end{array}$ \\
\hline 22 & 01546400 & Spring Creek at Houserville, $\mathrm{Pa}$. & $\begin{array}{l}40^{\circ} 50^{\prime} 01^{\prime \prime} \\
77^{\circ} 49^{\prime} 40^{\prime \prime}\end{array}$ & $\mathrm{RV}-\mathrm{am}-100$ & 76 & $\begin{array}{l}\text { From } 255 \mathrm{ft} \text { U.S. of station to } 745 \mathrm{ft} \text { D.S. of } \\
\text { station. Total length is } 1,000 \mathrm{ft} .\end{array}$ \\
\hline 23 & 01546500 & Spring Creek near Axemann, Pa. & $\begin{array}{l}40^{\circ} 53^{\prime} 23^{\prime \prime} \\
77^{\circ} 47^{\prime} 40^{\prime \prime}\end{array}$ & RV - am - 100 & 83 & $\begin{array}{l}\text { From } 860 \mathrm{ft} \text { U.S. of station to } 500 \mathrm{ft} \text { D.S. of } \\
\text { station. Total length is } 1,360 \mathrm{ft} .\end{array}$ \\
\hline 24 & 01547100 & Spring Creek at Milesburg, $\mathrm{Pa}$. & $\begin{array}{l}40^{\circ} 55^{\prime} 54^{\prime \prime} \\
77^{\circ} 47^{\prime} 13^{\prime \prime}\end{array}$ & RV - am - 100 & 78 & $\begin{array}{l}\text { From } 35 \mathrm{ft} \text { U.S. of station to } 1,285 \mathrm{ft} \text { D.S. of } \\
\text { station. Total length is } 1,320 \mathrm{ft} .\end{array}$ \\
\hline 25 & 01547700 & Marsh Creek at Blanchard, Pa. & $\begin{array}{l}41^{\circ} 03^{\prime} 34^{\prime \prime} \\
77^{\circ} 36^{\prime} 22^{\prime \prime}\end{array}$ & $\begin{array}{l}\text { RV - am - } 73 \\
\text { AP - alp - } 20 \\
A P-d v-7\end{array}$ & - & $\begin{array}{l}\text { From } 30 \mathrm{ft} \text { U.S. of station to } 970 \mathrm{ft} \text { D.S. of } \\
\text { station. Total length is } 1,000 \mathrm{ft} \text {. }\end{array}$ \\
\hline 26 & 01549500 & $\begin{array}{l}\text { Blockhouse Creek near English Center, } \\
\text { Pa. }\end{array}$ & $\begin{array}{l}41^{\circ} 28^{\prime} 25^{\prime \prime} \\
77^{\circ} 13^{\prime} 52^{\prime \prime}\end{array}$ & $\begin{array}{l}\text { AP - glp - } 35 \\
\text { AP - ghp - } 34 \\
\text { AP - dv - } 31\end{array}$ & - & $\begin{array}{l}\text { From } 1,347 \mathrm{ft} \text { U.S. of station to } 45 \mathrm{ft} \text { D.S. of } \\
\text { station. Total length is } 1,392 \mathrm{ft} \text {. }\end{array}$ \\
\hline 27 & 01550000 & Lycoming Creek near Trout Run, Pa. & $\begin{array}{l}41^{\circ} 25^{\prime} 06^{\prime \prime} \\
77^{\circ} 01^{\prime} 59^{\prime \prime}\end{array}$ & $\begin{array}{l}\text { AP - ghp - } 72 \\
\text { AP - glp - } 26 \\
\text { AP - dv - } 2\end{array}$ & - & $\begin{array}{l}\text { From } 180 \mathrm{ft} \text { U.S. of station to } 1,920 \mathrm{ft} \text { D.S. of } \\
\text { station. Total length is } 2,100 \mathrm{ft} \text {. }\end{array}$ \\
\hline 28 & 01552500 & Muncy Creek near Sonestown, Pa. & $\begin{array}{l}41^{\circ} 21^{\prime} 25^{\prime \prime} \\
76^{\circ} 32^{\prime} 06^{\prime \prime}\end{array}$ & AP - ghp - 100 & - & $\begin{array}{l}\text { From } 229 \mathrm{ft} \mathrm{U.S.} \mathrm{of} \mathrm{station} \mathrm{to} 1,611 \mathrm{ft} \text { D.S. of } \\
\text { station. Total length is } 1,840 \mathrm{ft} \text {. }\end{array}$ \\
\hline 29 & 01553700 & $\begin{array}{l}\text { Chillisquaque Creek at Washingtonville, } \\
\text { Pa. }\end{array}$ & $\begin{array}{l}41^{\circ} 03^{\prime} 42^{\prime \prime} \\
76^{\circ} 40^{\prime} 50^{\prime \prime}\end{array}$ & RV - am - 100 & 6 & $\begin{array}{l}\text { From station to } 1,867 \mathrm{ft} \text { D.S. of station. Total } \\
\text { length is } 1,867 \mathrm{ft} \text {. }\end{array}$ \\
\hline
\end{tabular}


Table 1. Physiographic characteristics and percentage of carbonate bedrock underlying watersheds of streamflow-gaging stations and associated stream reaches assessed for regional-curve development, Pennsylvania and selected areas of Maryland._-Continued

[—, no carbonate bedrock; ft, feet; St., Street; D.S., downstream; U.S., upstream; RV, Ridge and Valley Province; am, Appalachian Mountain Section; gv, Great Valley Section; AP,

Appalachian Plateaus Province; alm, Allegheny Mountain Section; alp, Allegheny Plateau Section; dv, Deep Valleys Section; ghp, Glaciated High Plateau Section; glp, Glaciated Low Plateau Section; gpp, Glaciated Pittsburgh Plateau Section; gpop, Glaciated Pocono Plateau Section; hp, High Plateau Section; plp, Pittsburgh Low Plateau Section; P, Piedmont Province; gnl, Gettysburg-Newark Lowland Section; 1, Lowland Section; u, Upland Section; BR, Blue Ridge Province; sm, South Mountain Section; CL, Central Lowland Province; el, Eastern Lake Section; NE, New England Province; rp, Reading Prong Section]

\begin{tabular}{|c|c|c|c|c|c|c|}
\hline $\begin{array}{l}\text { Cross- } \\
\text { reference } \\
\text { identification } \\
\text { number }\end{array}$ & $\begin{array}{c}\text { U.S. } \\
\text { Geological } \\
\text { Survey } \\
\text { Station } \\
\text { identifi- } \\
\text { cation } \\
\text { number }\end{array}$ & Station name & $\begin{array}{l}\text { Latitude/ } \\
\text { longitude }\end{array}$ & $\begin{array}{l}\text { Physiographic unit } \\
\text { (percentage } \\
\text { underlying total } \\
\text { watershed area) }{ }^{2}\end{array}$ & $\begin{array}{c}\text { Percentage } \\
\text { of } \\
\text { watershed } \\
\text { underlain by } \\
\text { carbonate } \\
\text { bedrock }^{3}\end{array}$ & Reach location \\
\hline 30 & 01555500 & $\begin{array}{l}\text { East Mahantango Creek near Dalmatia, } \\
\text { Pa. }\end{array}$ & $\begin{array}{l}40^{\circ} 36^{\prime} 40^{\prime \prime} \\
76^{\circ} 54^{\prime} 44^{\prime \prime}\end{array}$ & RV - am - 100 & - & $\begin{array}{l}\text { From } 1,067 \mathrm{ft} \text { U.S. of station to } 323 \mathrm{ft} \mathrm{D.S.} \mathrm{of} \\
\text { station. Total length is } 1,390 \mathrm{ft} \text {. }\end{array}$ \\
\hline 31 & 01565000 & Kishacoquillas Creek at Reedsville, $\mathrm{Pa}$. & $\begin{array}{l}40^{\circ} 39^{\prime} 17^{\prime \prime} \\
77^{\circ} 35^{\prime} 00^{\prime \prime}\end{array}$ & $\mathrm{RV}-\mathrm{am}-100$ & 25 & $\begin{array}{l}\text { From } 1,337 \mathrm{ft} \text { U.S. of station to } 18 \mathrm{ft} \text { D.S. of } \\
\text { station. Total length is } 1,355 \mathrm{ft} .\end{array}$ \\
\hline 32 & 01566000 & Tuscarora Creek near Port Royal, Pa. & $\begin{array}{l}40^{\circ} 30^{\prime} 55^{\prime \prime} \\
77^{\circ} 25^{\prime} 10^{\prime \prime}\end{array}$ & RV - am - 100 & 12 & $\begin{array}{l}\text { From } 1,367 \mathrm{ft} \text { U.S. of station to } 645 \mathrm{ft} \text { D.S of } \\
\text { station. Total length is } 2,012 \mathrm{ft} .\end{array}$ \\
\hline 33 & 01567500 & Bixler Run near Loysville, Pa. & $\begin{array}{l}40^{\circ} 22^{\prime} 15^{\prime \prime} \\
77^{\circ} 24^{\prime} 09^{\prime \prime}\end{array}$ & RV - am - 100 & 19 & $\begin{array}{l}\text { From } 608 \mathrm{ft} \text { U.S. of station to } 292 \mathrm{ft} \text { D.S. of } \\
\text { station. Total length is } 900 \mathrm{ft} \text {. }\end{array}$ \\
\hline 34 & 01568000 & Sherman Creek at Shermans Dale, Pa. & $\begin{array}{l}40^{\circ} 19^{\prime} 24^{\prime \prime} \\
77^{\circ} 10^{\prime} 09^{\prime \prime}\end{array}$ & RV - am - 100 & 12 & $\begin{array}{l}\text { From } 882 \mathrm{ft} \text { U.S of station to } 168 \mathrm{ft} \text { D.S. of } \\
\text { station. Total length is } 1,050 \mathrm{ft} \text {. }\end{array}$ \\
\hline 35 & 01569340 & Newburg Run at Newburg, Pa. & $\begin{array}{l}40^{\circ} 07^{\prime} 40^{\prime \prime} \\
77^{\circ} 32^{\prime} 50^{\prime \prime}\end{array}$ & $\begin{array}{l}R V-g v-92 \\
R V-a m-8\end{array}$ & - & $\begin{array}{l}\text { From station to } 571 \mathrm{ft} \text { D.S. Total length is } \\
571 \mathrm{ft} \text {. }\end{array}$ \\
\hline 36 & 01571500 & $\begin{array}{l}\text { Yellow Breeches Creek near Camp Hill, } \\
\text { Pa. }\end{array}$ & $\begin{array}{l}40^{\circ} 13^{\prime} 29^{\prime \prime} \\
76^{\circ} 53^{\prime} 54^{\prime \prime}\end{array}$ & $\begin{array}{l}R V-g v-41 \\
B R-s m-40 \\
P-g n l-19\end{array}$ & 34 & $\begin{array}{l}\text { From } 117 \mathrm{ft} \mathrm{U.S.} \mathrm{of} \mathrm{station} \mathrm{to} 1,547 \mathrm{ft} \text { D.S. of } \\
\text { station. Total length is } 1,664 \mathrm{ft} \text {. }\end{array}$ \\
\hline 37 & 01573160 & Quittapahilla Creek near Bellegrove, Pa. & $\begin{array}{l}40^{\circ} 20^{\prime} 34^{\prime \prime} \\
76^{\circ} 33^{\prime} 46^{\prime \prime}\end{array}$ & $\begin{array}{l}R V-g v-87 \\
P-\text { gnl }-13\end{array}$ & 74 & $\begin{array}{l}\text { From } 10 \mathrm{ft} \text { U.S. of station to } 1,135 \mathrm{ft} \text { D.S. of } \\
\text { station. Total length is } 1,145 \mathrm{ft} \text {. }\end{array}$ \\
\hline 38 & 01613050 & Tonoloway Creek near Needmore, Pa. & $\begin{array}{l}39^{\circ} 53^{\prime} 54^{\prime \prime} \\
78^{\circ} 07^{\prime} 57^{\prime \prime}\end{array}$ & $\mathrm{RV}-\mathrm{am}-100$ & - & $\begin{array}{l}\text { From } 594 \mathrm{ft} \text { U.S. of station to } 431 \mathrm{ft} \text { D.S. of } \\
\text { station. Total length is } 1,025 \mathrm{ft} .\end{array}$ \\
\hline 39 & 01617800 & Marsh Run at Grimes, Md. ${ }^{4}$ & $\begin{array}{l}39^{\circ} 30^{\prime} 52^{\prime \prime} \\
77^{\circ} 46^{\prime} 38^{\prime \prime}\end{array}$ & $\mathrm{RV}-\mathrm{gv}-100$ & 100 & $\begin{array}{l}\text { From } 253 \mathrm{ft} \text { U.S. of station to } 288 \mathrm{ft} \text { D.S. of } \\
\text { station. Total length is } 541 \mathrm{ft} .\end{array}$ \\
\hline 40 & 03011800 & Kinzua Creek near Guffey, Pa. & $\begin{array}{l}41^{\circ} 45^{\prime} 59^{\prime \prime} \\
78^{\circ} 43^{\prime} 08^{\prime \prime}\end{array}$ & AP - hp - 100 & - & $\begin{array}{l}\text { From } 107 \mathrm{ft} \text { U.S. of station to } 1,323 \mathrm{ft} \text { D.S. of } \\
\text { station. Total length is } 1,430 \mathrm{ft} .\end{array}$ \\
\hline 41 & 03021410 & $\begin{array}{l}\text { West Branch French Creek near Lowville, } \\
\text { Pa. }\end{array}$ & $\begin{array}{l}42^{\circ} 04^{\prime} 54^{\prime \prime} \\
79^{\circ} 51^{\prime} 02^{\prime \prime}\end{array}$ & AP - gpp - 100 & - & $\begin{array}{l}\text { From } 847 \mathrm{ft} \text { U.S. of station to } 153 \mathrm{ft} \text { D.S. of } \\
\text { station. Total length is } 1,000 \mathrm{ft} .\end{array}$ \\
\hline 42 & 03022540 & Woodcock Creek at Blooming Valley, Pa. & $\begin{array}{l}41^{\circ} 41^{\prime} 26^{\prime \prime} \\
80^{\circ} 02^{\prime} 26^{\prime \prime}\end{array}$ & AP - gpp - 100 & - & $\begin{array}{l}\text { From } 974 \mathrm{ft} \text { U.S. of station to } 240 \mathrm{ft} \text { D.S. of } \\
\text { station. Total length is } 1,214 \mathrm{ft} .\end{array}$ \\
\hline 43 & 03026500 & Sevenmile Run near Rasselas, $\mathrm{Pa}$. & $\begin{array}{l}41^{\circ} 37^{\prime} 52^{\prime \prime} \\
78^{\circ} 34^{\prime} 37^{\prime \prime}\end{array}$ & AP - hp - 100 & - & $\begin{array}{l}\text { From } 757 \mathrm{ft} \text { U.S. of station to } 45 \mathrm{ft} \text { D.S. of } \\
\text { station. Total length is } 802 \mathrm{ft} .\end{array}$ \\
\hline 44 & 03028000 & West Branch Clarion River at Wilcox, Pa. & $\begin{array}{l}41^{\circ} 34^{\prime} 45^{\prime \prime} \\
78^{\circ} 41^{\prime} 22^{\prime \prime}\end{array}$ & AP - hp - 100 & - & $\begin{array}{l}\text { From } 28 \mathrm{ft} \text { U.S. of station to } 1,342 \mathrm{ft} \text { D.S. of } \\
\text { station. Total length is } 1,370 \mathrm{ft} .\end{array}$ \\
\hline
\end{tabular}


Table 1. Physiographic characteristics and percentage of carbonate bedrock underlying watersheds of streamflow-gaging stations and associated stream reaches assessed for regional-curve development, Pennsylvania and selected areas of Maryland.-Continued

[—, no carbonate bedrock; ft, feet; St., Street; D.S., downstream; U.S., upstream; RV, Ridge and Valley Province; am, Appalachian Mountain Section; gv, Great Valley Section; AP, Appalachian Plateaus Province; alm, Allegheny Mountain Section; alp, Allegheny Plateau Section; dv, Deep Valleys Section; ghp, Glaciated High Plateau Section; glp, Glaciated Low Plateau Section; gpp, Glaciated Pittsburgh Plateau Section; gpop, Glaciated Pocono Plateau Section; hp. High Plateau Section; plp, Pittsburgh Low Plateau Section; P, Piedmont Province; gnl, Gettysburg-Newark Lowland Section; 1 , Lowland Section; u, Upland Section; BR, Blue Ridge Province; sm, South Mountain Section; CL, Central Lowland Province; el, Eastern Lake Section; NE, New England Province; rp, Reading Prong Section]

\begin{tabular}{|c|c|c|c|c|c|c|}
\hline $\begin{array}{l}\text { Cross- } \\
\text { reference } \\
\text { identification } \\
\text { number }\end{array}$ & $\begin{array}{c}\text { U.S. } \\
\text { Geological } \\
\text { Survey } \\
\text { Station } \\
\text { identifi- } \\
\text { cation } \\
\text { number }\end{array}$ & Station name & $\begin{array}{l}\text { Latitude/ } \\
\text { longitude }^{1}\end{array}$ & $\begin{array}{l}\text { Physiographic unit } \\
\text { (percentage } \\
\text { underlying total } \\
\text { watershed area) }^{2}\end{array}$ & $\begin{array}{c}\text { Percentage } \\
\text { of } \\
\text { watershed } \\
\text { underlain by } \\
\text { carbonate } \\
\text { bedrock }^{3}\end{array}$ & Reach location \\
\hline 45 & 03034500 & Little Mahoning Creek at McCormick, Pa. & $\begin{array}{l}40^{\circ} 50^{\prime} 10^{\prime \prime} \\
79^{\circ} 06^{\prime} 37^{\prime \prime}\end{array}$ & AP - plp - 100 & - & $\begin{array}{l}\text { From } 277 \mathrm{ft} \text { U.S. of station to } 1,823 \mathrm{ft} \text { D.S. of } \\
\text { station. Total length is } 2,100 \mathrm{ft} \text {. }\end{array}$ \\
\hline 46 & 03039925 & North Fork Bens Creek at North Fork, Pa. & $\begin{array}{l}40^{\circ} 15^{\prime} 58^{\prime \prime} \\
79^{\circ} 01^{\prime} 01^{\prime \prime}\end{array}$ & AP - alm - 100 & - & $\begin{array}{l}\text { From } 751 \mathrm{ft} \mathrm{U.S.} \mathrm{of} \mathrm{station} \mathrm{to} \mathrm{station.} \mathrm{Total} \\
\text { length is } 751 \mathrm{ft} \text {. }\end{array}$ \\
\hline 47 & 03049000 & Buffalo Creek near Freeport, $\mathrm{Pa}$. & $\begin{array}{l}40^{\circ} 42^{\prime} 57^{\prime \prime} \\
79^{\circ} 41^{\prime} 59^{\prime \prime}\end{array}$ & AP - plp - 100 & - & $\begin{array}{l}\text { From } 1,711 \mathrm{ft} \text { U.S. of station to } 24 \mathrm{ft} \text { D.S of } \\
\text { station. Total length is } 1,735 \mathrm{ft} \text {. }\end{array}$ \\
\hline 48 & 03049800 & Little Pine Creek near Etna, Pa. & $\begin{array}{l}40^{\circ} 31^{\prime} 13^{\prime \prime} \\
79^{\circ} 56^{\prime} 18^{\prime \prime}\end{array}$ & AP - plp - 100 & - & $\begin{array}{l}\text { From } 552 \mathrm{ft} \text { U.S. of station to } 1,007 \mathrm{ft} \text { D.S. of } \\
\text { station. Total length is } 1,559 \mathrm{ft} \text {. }\end{array}$ \\
\hline 49 & 03072880 & Browns Creek near Nineveh, Pa. & $\begin{array}{l}39^{\circ} 56^{\prime} 27^{\prime \prime} \\
80^{\circ} 17^{\prime} 21^{\prime \prime}\end{array}$ & AP - plp - 100 & - & $\begin{array}{l}\text { From station to } 1,190 \mathrm{ft} \text { D.S. Total length is } \\
1,190 \mathrm{ft} \text {. }\end{array}$ \\
\hline 50 & 03080000 & Laurel Hill Creek at Ursina, Pa. & $\begin{array}{l}39^{\circ} 49^{\prime} 13^{\prime \prime} \\
79^{\circ} 19^{\prime} 18^{\prime \prime}\end{array}$ & AP - alm - 100 & - & $\begin{array}{l}\text { From } 290 \mathrm{ft} \text { U.S. of station to } 1,300 \mathrm{ft} \text { D.S. of } \\
\text { station. Total length is } 1,590 \mathrm{ft} \text {. }\end{array}$ \\
\hline 51 & 03102500 & Little Shenango River at Greenville, Pa. & $\begin{array}{l}41^{\circ} 25^{\prime} 19^{\prime \prime} \\
80^{\circ} 22^{\prime} 35^{\prime \prime}\end{array}$ & AP - gpp - 100 & - & $\begin{array}{l}\text { From } 20 \mathrm{ft} \text { U.S. of station to } 1,210 \mathrm{ft} \text { D.S. of } \\
\text { station. Total length is } 1,230 \mathrm{ft} \text {. }\end{array}$ \\
\hline 52 & 04213075 & Brandy Run near Girard, Pa. & $\begin{array}{l}41^{\circ} 59^{\prime} 31^{\prime \prime} \\
80^{\circ} 17^{\prime} 29^{\prime \prime}\end{array}$ & CL - el - 100 & - & $\begin{array}{l}\text { From } 910 \mathrm{ft} \mathrm{U.S.} \mathrm{of} \mathrm{station} \mathrm{to} 15 \mathrm{ft} \text { D.S. of } \\
\text { station. Total length is } 925 \mathrm{ft} \text {. }\end{array}$ \\
\hline${ }^{5} \mathrm{~W} 1$ & 01471980 & Manatawny Creek near Pottstown, Pa. & $\begin{array}{l}40^{\circ} 16^{\prime} 22^{\prime \prime} \\
75^{\circ} 40^{\prime} 49^{\prime \prime}\end{array}$ & $\begin{array}{l}\text { NE - rp - } 60 \\
R V-\text { gv }-28 \\
P-\text { gnl }-12\end{array}$ & 26 & $\begin{array}{l}\text { From } 1,536 \mathrm{ft} \text { U.S. of station to } 108 \mathrm{ft} \text { D.S. of } \\
\text { station. Total length is } 1,644 \mathrm{ft} \text {. }\end{array}$ \\
\hline W2 & 01472157 & French Creek near Phoenixville, $\mathrm{Pa}$. & $\begin{array}{l}40^{\circ} 09^{\prime} 05^{\prime \prime} \\
75^{\circ} 36^{\prime} 06^{\prime \prime}\end{array}$ & $\begin{array}{l}P-\text { gnl }-51 \\
P-u-49\end{array}$ & - & $\begin{array}{l}\text { From } 136 \mathrm{ft} \text { U.S. of station to } 1,046 \mathrm{ft} \text { D.S. of } \\
\text { station. Total length is } 1,182 \mathrm{ft} \text {. }\end{array}$ \\
\hline W3 & 01472198 & Perkiomen Creek at East Greenville, Pa. & $\begin{array}{l}40^{\circ} 23^{\prime} 38^{\prime \prime} \\
75^{\circ} 30^{\prime} 57^{\prime \prime}\end{array}$ & $\begin{array}{l}\text { NE - rp - } 60 \\
P \text { - gnl - } 38 \\
\text { RV - gv - } 2\end{array}$ & 3 & $\begin{array}{l}\text { From station to } 1,000 \mathrm{ft} \text { D.S. of station. Total } \\
\text { length is } 1,000 \mathrm{ft} .\end{array}$ \\
\hline W4 & 01472199 & $\begin{array}{l}\text { West Branch Perkiomen Creek at Hille- } \\
\text { gas, Pa. }\end{array}$ & $\begin{array}{l}40^{\circ} 22^{\prime} 26^{\prime \prime} \\
75^{\circ} 31^{\prime} 22^{\prime \prime}\end{array}$ & $P-u-100$ & 4 & $\begin{array}{l}\text { From } 1,184 \mathrm{ft} \text { U.S. of station to } 316 \mathrm{ft} \text { D.S. of } \\
\text { station. Total length is } 1,500 \mathrm{ft} \text {. }\end{array}$ \\
\hline${ }^{6} \mathrm{C} 5$ & 01475850 & Crum Creek near Newtown Square, Pa. & $\begin{array}{l}39^{\circ} 58^{\prime} 35^{\prime \prime} \\
75^{\circ} 26^{\prime} 13^{\prime \prime}\end{array}$ & $P-u-100$ & - & $\begin{array}{l}\text { From } 891 \mathrm{ft} \mathrm{U.S.} \mathrm{of} \mathrm{station} \mathrm{to} \mathrm{station.} \mathrm{Total} \\
\text { length is } 891 \mathrm{ft} \text {. }\end{array}$ \\
\hline C6 & 01477000 & Chester Creek near Chester, Pa. & $\begin{array}{l}39^{\circ} 52^{\prime} 10^{\prime \prime} \\
75^{\circ} 24^{\prime} 30^{\prime \prime}\end{array}$ & $P-u-100$ & - & $\begin{array}{l}\text { From station to } 900 \mathrm{ft} \text { D.S. of station. Total } \\
\text { length is } 900 \mathrm{ft} \text {. }\end{array}$ \\
\hline
\end{tabular}


Table 1. Physiographic characteristics and percentage of carbonate bedrock underlying watersheds of streamflow-gaging stations and associated stream reaches assessed for regional-curve development, Pennsylvania and selected areas of Maryland._-Continued

[—, no carbonate bedrock; ft, feet; St., Street; D.S., downstream; U.S., upstream; RV, Ridge and Valley Province; am, Appalachian Mountain Section; gv, Great Valley Section; AP,

Appalachian Plateaus Province; alm, Allegheny Mountain Section; alp, Allegheny Plateau Section; dv, Deep Valleys Section; ghp, Glaciated High Plateau Section; glp, Glaciated Low Plateau Section; gpp, Glaciated Pittsburgh Plateau Section; gpop, Glaciated Pocono Plateau Section; hp, High Plateau Section; plp, Pittsburgh Low Plateau Section; P, Piedmont Province; gnl, Gettysburg-Newark Lowland Section; 1, Lowland Section; u, Upland Section; BR, Blue Ridge Province; sm, South Mountain Section; CL, Central Lowland Province; el, Eastern Lake Section; NE, New England Province; rp, Reading Prong Section]

\begin{tabular}{|c|c|c|c|c|c|c|}
\hline $\begin{array}{c}\text { Cross- } \\
\text { reference } \\
\text { identification } \\
\text { number }\end{array}$ & $\begin{array}{c}\text { U.S. } \\
\text { Geological } \\
\text { Survey } \\
\text { Station } \\
\text { identifi- } \\
\text { cation } \\
\text { number }\end{array}$ & Station name & $\begin{array}{l}\text { Latitude/ } \\
\text { longitude }^{1}\end{array}$ & $\begin{array}{l}\text { Physiographic unit } \\
\text { (percentage } \\
\text { underlying total } \\
\text { watershed area) }\end{array}$ & $\begin{array}{c}\text { Percentage } \\
\text { of } \\
\text { watershed } \\
\text { underlain by } \\
\text { carbonate } \\
\text { bedrock }^{3}\end{array}$ & Reach location \\
\hline $\mathrm{C} 7$ & 01480300 & $\begin{array}{l}\text { West Branch Brandywine Creek near } \\
\text { Honey Brook, Pa. }\end{array}$ & $\begin{array}{l}40^{\circ} 04^{\prime} 22^{\prime \prime} \\
75^{\circ} 51^{\prime} 40^{\prime \prime}\end{array}$ & $\mathrm{P}-\mathrm{u}-100$ & 3 & $\begin{array}{l}\text { From } 917 \mathrm{ft} \text { U.S. of station to } 71 \mathrm{ft} \text { D.S. of } \\
\text { station. Total length is } 988 \mathrm{ft} .\end{array}$ \\
\hline $\mathrm{C} 8$ & 01480500 & $\begin{array}{l}\text { West Branch Brandywine Creek at } \\
\text { Coatesville, Pa. }\end{array}$ & $\begin{array}{l}39^{\circ} 59^{\prime} 08^{\prime \prime} \\
75^{\circ} 49^{\prime} 40^{\prime \prime}\end{array}$ & $P-u-100$ & 1 & $\begin{array}{l}\text { From station to } 690 \mathrm{ft} \text { D.S. of station. Total } \\
\text { length is } 690 \mathrm{ft} \text {. }\end{array}$ \\
\hline W9 & 01480610 & Sucker Run near Coatesville, Pa. & $\begin{array}{l}39^{\circ} 58^{\prime} 20^{\prime \prime} \\
75^{\circ} 51^{\prime} 03^{\prime \prime}\end{array}$ & $\begin{array}{l}P-u-75 \\
P-1-25\end{array}$ & 36 & $\begin{array}{l}\text { From station to } 280 \mathrm{ft} \text { D.S. of station. Total } \\
\text { length is } 280 \mathrm{ft} \text {. }\end{array}$ \\
\hline $\mathrm{C} 10$ & 01480617 & $\begin{array}{l}\text { West Branch Brandywine Creek at } \\
\text { Modena, Pa. }\end{array}$ & $\begin{array}{l}39^{\circ} 57^{\prime} 42^{\prime \prime} \\
75^{\circ} 48^{\prime} 06^{\prime \prime}\end{array}$ & $\begin{array}{l}P-u-96 \\
P-1-4\end{array}$ & 6 & $\begin{array}{l}\text { From station to } 900 \mathrm{ft} \text { D.S. of station. Total } \\
\text { length is } 900 \mathrm{ft} \text {. }\end{array}$ \\
\hline $\mathrm{C} 11$ & 01578200 & Conowingo Creek near Buck, Pa. & $\begin{array}{l}39^{\circ} 50^{\prime} 35^{\prime \prime} \\
76^{\circ} 11^{\prime} 45^{\prime \prime}\end{array}$ & $P-u-100$ & - & $\begin{array}{l}\text { From } 259 \mathrm{ft} \text { U.S. of station to } 480 \mathrm{ft} \text { D.S. of } \\
\text { station. Total length is } 739 \mathrm{ft} \text {. }\end{array}$ \\
\hline${ }^{7} \mathrm{C} 12$ & 01586210 & Beaver Run near Finksburg, Md. & $\begin{array}{l}39^{\circ} 29^{\prime} 22^{\prime \prime} \\
76^{\circ} 54^{\prime} 12^{\prime \prime}\end{array}$ & $P-u-100$ & - & $\begin{array}{l}\text { From station to } 975 \mathrm{ft} \text { D.S. of station. Total } \\
\text { length is } 975 \mathrm{ft} \text {. }\end{array}$ \\
\hline${ }^{7} \mathrm{C} 13$ & 01586610 & Morgan Run near Louisville, Md. & $\begin{array}{l}39^{\circ} 27^{\prime} 07^{\prime \prime} \\
76^{\circ} 57^{\prime} 20^{\prime \prime}\end{array}$ & $P-u-100$ & - & $\begin{array}{l}\text { From } 802 \mathrm{ft} \text { U.S. of station to } 458 \mathrm{ft} \text { D.S. of } \\
\text { station. Total length is } 1,260 \mathrm{ft} \text {. }\end{array}$ \\
\hline${ }^{7} \mathrm{~W} 14$ & 01639500 & Big Pipe Creek at Bruceville, Md. & $\begin{array}{l}39^{\circ} 36^{\prime} 45^{\prime \prime} \\
77^{\circ} 14^{\prime} 10^{\prime \prime}\end{array}$ & $\begin{array}{l}P-u-68 \\
P-g n l-32\end{array}$ & - & $\begin{array}{l}\text { From } 854 \mathrm{ft} \text { U.S. of station to } 339 \mathrm{ft} \text { D.S. of } \\
\text { station. Total length is } 1,193 \mathrm{ft} \text {. }\end{array}$ \\
\hline
\end{tabular}

\footnotetext{
${ }^{1}$ Referenced to the North American Datum of 1927
}

${ }^{2}$ Computed from coverages published by Pennsylvania Department of Conservation and Natural Resources (1995) and Langland and others (1995).

${ }^{3}$ Percentage of watershed underlain by carbonate bedrock determined from Pennsylvania Department of Conservation and Natural Resources (2000b)

${ }^{4}$ All data provided by Edward Doheny, U.S. Geological Survey, written commun., 2004.

5 "W" indicates that data were originally published in White (2001).

6“"C" indicates that data were originally published in Cinotto (2003).

${ }^{7}$ All data provided by R.W. James, Jr., U.S. Geological Survey, oral commun., 2001. 
A longitudinal survey of the thalweg, water surface, and bankfull-channel elevations was completed at each station along with cross-sectional surveys. At most stations, bankfullchannel geometry was determined by averaging bankfull area, width, and mean depth determined by cross-sectional surveys at two riffles. Bankfull-channel geometry at stations W1 and W14 is based on only one cross section because the reach near these stations contained only one riffle. (See the Appendix for the location, photographs, and data associated with cross-sectional surveys in all stream reaches.)

Note that the bankfull-channel elevation as used here is considered to be the elevation of incipient flooding, the interface between the active channel and floodplain. All surveys were referenced to the datum established for gaging streamflow (fig. 2). Surveyed elevations were recorded to the hundredth of a foot in accordance with the accuracy of the equipment and survey techniques. After the bankfull channel was selected, the water-surface elevation that would occur in the bankfull channel was used to determine the bankfull discharge at most stations. Specifically, the bankfull discharge was determined by comparing the elevation of the bankfull channel (fig. 2) to the rating at each station.

At some stations, the bankfull discharge could not be determined from the rating because of large changes in streamchannel elevations between the streamflow-gaging station and stream reach along which the bankfull channel was surveyed. This situation generally was caused by $10-20 \mathrm{ft}$ control structures that impounded water for 1,000 to $2,000 \mathrm{ft}$ upstream. The differences in elevation occasionally resulted in a "disconnect" between bankfull features within the impounded reaches and the downstream reaches. In such cases, indicators of the bankfull channel within the impoundments were created by different flow regimes than in the downstream reaches and were not used in conjunction with the ratings to determine bankfull discharges. Instead, channel geometry was determined at the two cross sections as normal, and slope-conveyance methods described in Rosgen (1996) were used as an alternative for determining bankfull discharge. Discharges computed at each surveyed cross section were averaged to compute the bankfull discharge used for development of regional curves.

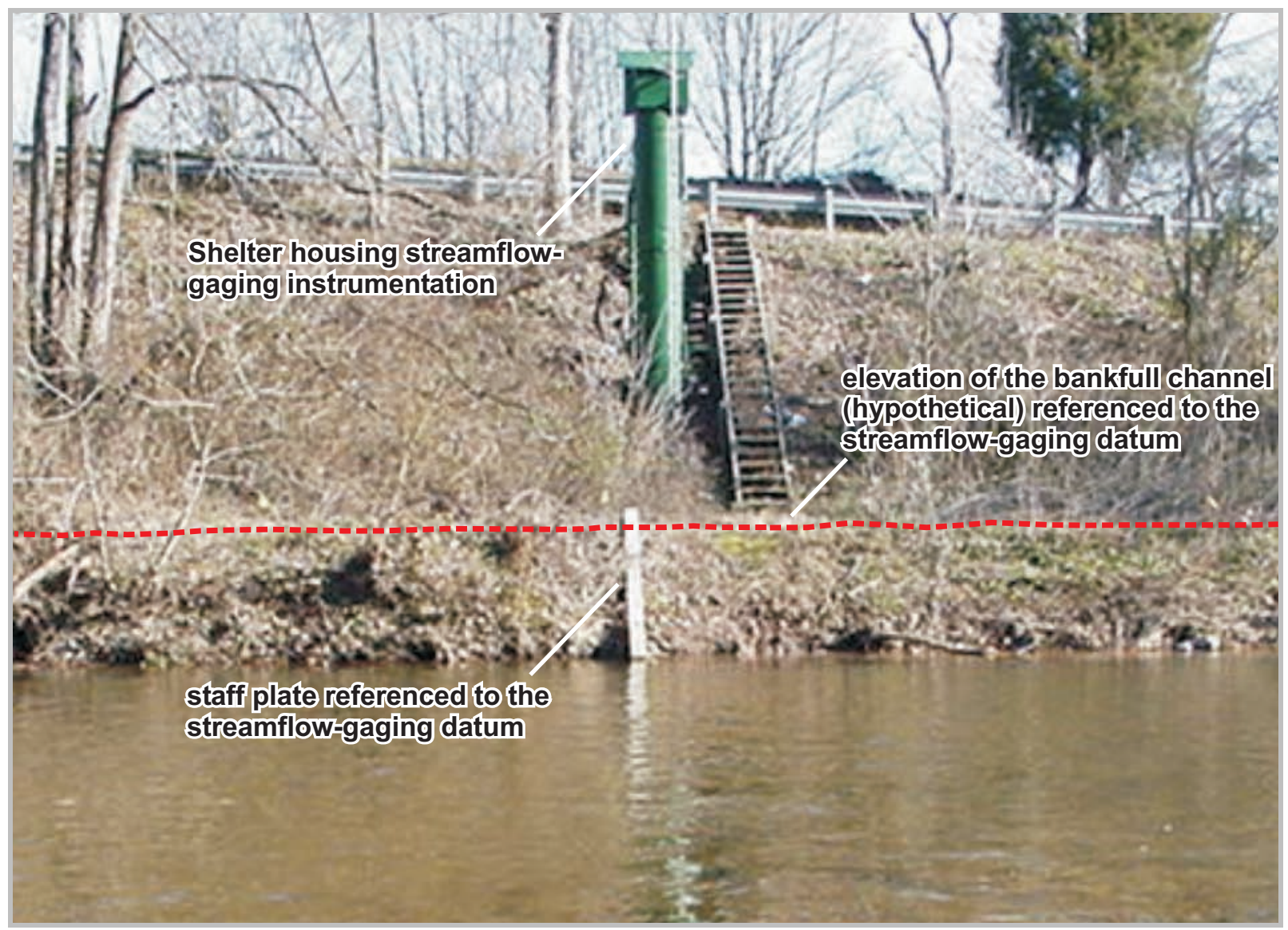

Figure 2. Streamflow-gaging station and hypothetical example of a bankfull-channel elevation used for development of regional curves in Pennsylvania and selected areas of Maryland. Photograph taken by Kirk E. White, U.S. Geological Survey, January 2000. 
Historical cross-sectional channel geometry data collected during routine streamflow measurements at each station were helpful in identifying the bankfull channel at many but not all stations. Cross-sectional geometry is most useful for determination of the bankfull channel when one measurement section is located near a riffle and is used over a range of water surface elevations (including the bankfull elevation). Most USGS streamflow-measurement sections are selected to provide accurate velocity and area determinations for the computation of discharge. Thus, the location of the measurement section may vary depending on the water-surface elevation. The measuring section may be in slower pool-dominated sections and not in riffles as required by most fluvial-geomorphology computations. Changes in the location of the measurement section at a given station diminishes the usefulness of geometry measurements for selection of the bankfull channel because of scatter and the inability to determine exactly where the measurement was made. Therefore, narrative descriptions that accompany each streamflow measurement were reviewed to determine if channel geometry at the measurement section may be helpful for identification of the bankfull channel. When appropriate, these data were used to aid in identification of the bankfull channel.

\section{Statistical Procedures}

Statistical procedures that test for differences in regional curves between the three major physiographic provinces provide a means to determine if there is justification for separate relations or if more powerful relations can be achieved by combining data from some or all of the provinces. For example, if regional curves for the Piedmont and Ridge and Valley Physiographic Provinces have the same slope and intercept as the corresponding relations in the Appalachian Plateaus Physiographic Province, combination of the data across all three provinces will result in development of more robust curves. In contrast, a significant difference in slope or intercept of regional curves between physiographic provinces would indicate that separate curves are appropriate. The analysis of differences in slope and intercept due solely to physiography is complicated by other factors like carbonate bedrock and glaciation that may cause variability in bankfull characteristics. As a result, the influence of carbonate bedrock and glaciation is analyzed along with physiography in the statistical procedures described below.

A total of 63 stations were first assigned to either the Piedmont, Ridge and Valley, or Appalachian Plateaus Physiographic Province according to which province underlays the maximum percentage of the watershed area (table 1). Note that stations 52, W1, and W3 are not included because the maximum percentage of watershed area is underlain by the Central Lowland or New England Physiographic Provinces (table 1). The percentage of each physiographic unit underlying each watershed was determined with a GIS consisting of coverages from Pennsylvania Department of Conservation and Natural Resources (1995) and Langland and others (1995). Analysis of Covariance (ANCOVA), following procedures described by
SAS Institute, Inc. (1990), was used to analyze for differences in the slope and intercept of regression lines developed for each of the three provinces.

Karst features in watersheds underlain by carbonate bedrock can lead to underflow and ground-water storage (Kochanov and Reese, 2003) that may alter the bankfull discharge and associated channel geometry regardless of physiography. Of the 63 stations assigned to the Piedmont, Ridge and Valley, or Appalachian Plateaus Physiographic Provinces, 23 are in watersheds underlain by carbonate bedrock ranging from 1 to 100 percent of the watershed area (table 1). To isolate the influence of physiography, the potential influence of carbonate bedrock was eliminated by performing a second ANCOVA on regressions developed with the 40 watersheds in the Piedmont, Ridge and Valley, or Appalachian Plateaus Physiographic Province that are not underlain by any carbonate bedrock (table 1).

The ANCOVA tests were structured to compare the slope and intercept of regressions developed for the Ridge and Valley and Piedmont Physiographic Provinces to the corresponding relations of the Appalachian Plateaus Physiographic Province. Hence, the regression for the Appalachian Plateaus Province represents a control condition. Note that the Ridge and Valley and Piedmont Physiographic Provinces are not directly compared against one another but some conclusions regarding differences between these two provinces may be inferred. For example, if the statistical evidence indicates that regressions for the Piedmont and Ridge and Valley Physiographic Provinces are the same as those for the Appalachian Plateaus Province, then it can be inferred that the relations for the Piedmont and Ridge and Valley Province are also the same. The null hypothesis for each ANCOVA test is that there is no difference in the slope or intercept of regression equations for the Piedmont and Ridge and Valley Physiographic Provinces compared to the Appalachian Plateaus Physiographic Province. The null hypothesis is rejected if the $\mathrm{p}$-value (probability that a difference occurs by chance) is less than or equal to 0.0500 .

Following the ANCOVA tests, percent carbonate, percent glaciated, and drainage area were entered as explanatory variables in multiple-regression models developed to estimate bankfull discharge. The purposes of the multiple-regression models are twofold; (1) to determine if percent carbonate, percent glaciated, and (or) drainage area explain a significant amount of variation in bankfull discharge, and (2) to evaluate if the multiple-regression model provides better estimates of bankfull discharge than a regional curve relating bankfull discharge to drainage area alone. Statistical diagnostics and estimated bankfull discharges over a range of drainage areas were used to compare the strength of the multiple-regression model to the regional curve. The null hypothesis of the multipleregression models is that the slope coefficient of each explanatory variable is zero. That is, none of the explanatory variables have any predictive value. The null hypothesis was rejected if the p-value (probability that the slope coefficient is different from zero due to chance) was equal to or less than 0.0500 . 
For the purposes of this study, there are five assumptions for one- and multiple-variable regression models. They are (1) the bankfull response variable is linearly or log-linearly related to the explanatory variable(s); (2) data used to create the model are representative of the data of interest; (3) the variance of regression residuals is constant (homoscedastic); (4) the residuals are independent; and (5) the residuals are normally distributed (Helsel and Hirsch, 2002). The necessity of satisfying these assumptions is determined by the purpose of the regression equation. Adherence to assumptions 1 through 4 is necessary to obtain the best unbiased estimates of bankfullchannel geometry and discharge (Helsel and Hirsch, 2002).

\section{Data Analyses for Regional Curves}

Two findings from the ANCOVA tests have implications for development of regional curves presented in this report: (1) carbonate bedrock influences the slope of selected regression equations (tables 2 and 3), and (2) when the influence of carbonate bedrock is eliminated, the weight of evidence supports combination of data across all physiographic provinces. When watersheds underlain by carbonate bedrock are included, the slope of the bankfull-discharge regression for the Ridge and Valley Physiographic Province is significantly different from the corresponding equation for the Appalachian Plateaus Physiographic Province (p-value $=0.0078$; table 2 ). After the influence of carbonate bedrock is eliminated, the slope of this regression steepens and no longer differs from the Appalachian Plateaus equation (p-value $=0.1861$; table 3 ).

The p-values from ANCOVA tests indicate that the bankfull-mean-depth regression for the Piedmont Physiographic Province may differ from the corresponding relation for the Appalachian Plateaus Physiographic Province even after the influence of carbonate bedrock is eliminated ( $p$-values $=0.0065$ and 0.0339 ; tables 2 and 3 , respectively). Note however, that the $\mathrm{p}$-value of 0.0339 is relatively close to the significance level of 0.0500 and that there are only seven stations to define the slope and intercept of the regression equations in table 3. The p-values assign a confidence level to the intercept and slope-coefficient estimates based on the values and spread of the data ( $\mathrm{N}=7$ stations), not so much on the number of stations. Here, the concern is whether the regression defined by these seven stations is representative of the regression that would be defined by all possible stations. With a small "N," the chances are greater that it is not. This uncertainty, the relatively weak evidence that the bankfull-mean-depth regressions for the Piedmont and Appalachian Plateaus Physiographic Provinces are different, and that regressions for other bankfull characteristics in the Piedmont are not significantly different from the Appalachian Plateaus Physiographic Province, suggest that little or no increase in confidence and power can be achieved by develop- ing separate bankfull-mean-depth regressions for each physiographic province.

Results of the multiple-regression models suggest that including percent carbonate and (or) percent glaciated as explanatory variables in addition to drainage area does not yield better estimates of bankfull discharge compared to estimates from regional curves. Percent glaciated was not significant ( $p$-value $=0.0664)$ and percent carbonate caused the model to estimate some values of bankfull discharge that were physically impossible, even though it explained a significant amount of variability ( $\mathrm{p}$-value $=<0.0001$ ). For example, a model using percent carbonate and drainage area as explanatory variables estimated negative bankfull discharges for small watersheds because the intercept term on percent carbonate was negative. This is a major shortcoming, particularly for the practitioner relying on estimates that are applicable to natural-stream design.

The relation between bankfull characteristics and percent carbonate is difficult to evaluate statistically because of karst features (Kochanov and Reese, 2003) and poor representation of small watersheds underlain by carbonate bedrock. ANCOVA and multiple-regression results both suggest that carbonate bedrock influences bankfull characteristics, but neither has provided definitive evidence on how to account for this influence when estimating bankfull characteristics. Treating percent carbonate as a continuous variable in a multiple-regression model failed because the model was prone to estimate negative values for small drainage areas as mentioned above. However, if the percentage at which carbonate bedrock tends to markedly influence bankfull characteristics can be determined, then percent carbonate could be handled as a class variable. That is, separate regional curves could be developed for two subsets of watersheds; those that are influenced by carbonate bedrock and those that are not.

To investigate this possibility, drainage area and percent carbonate were entered as explanatory variables in another multiple-regression model to estimate bankfull discharge from 25 stations in watersheds underlain by 1 to 100 percent carbonate bedrock (table 1). Note that stations in this model were selected based on the presence of carbonate bedrock alone regardless of physiography. Partial residuals for percent carbonate indicate that this model commonly over-estimates bankfull discharge (partial residuals are negative) when the watershed area underlain by carbonate bedrock is $>30$ percent (fig. 3). With the exception of three stations, the model underestimates bankfull discharge (partial residuals are positive) when carbonate bedrock is $\leq 30$ percent (fig. 3 ). 
Table 3. Statistical results from analysis of covariance of regressions relating bankfull-channel characteristics to drainage area in watersheds without carbonate bedrock in the Piedmont, Ridge and Valley, and Appalachian Plateaus Physiographic Provinces.

[N, number of stations; --, control]

\begin{tabular}{|c|c|c|c|c|c|c|c|c|c|}
\hline \multirow{3}{*}{$\begin{array}{c}\text { Physiographic } \\
\text { Province }\end{array}$} & \multirow{3}{*}{$\mathbf{N}$} & \multirow{2}{*}{\multicolumn{2}{|c|}{$\begin{array}{c}\begin{array}{c}\text { Bankfull cross-sectional } \\
\text { area }\end{array} \\
p \text {-value }\end{array}$}} & \multirow{2}{*}{\multicolumn{2}{|c|}{$\begin{array}{c}\text { Bankfull discharge } \\
\text { p-value }\end{array}$}} & \multicolumn{2}{|c|}{ Bankfull width } & \multicolumn{2}{|c|}{ Bankfull mean depth } \\
\hline & & & & & & & & \multicolumn{2}{|c|}{ p-value } \\
\hline & & Intercept & Slope & Intercept & Slope & Intercept & Slope & Intercept & Slope \\
\hline Piedmont & 7 & 0.4449 & 0.0592 & 0.7702 & 0.2292 & 0.8772 & 0.4855 & 0.6687 & 0.0339 \\
\hline Ridge and Valley & 9 & .3294 & .8492 & .8476 & .1861 & .5675 & .1037 & .9314 & .6074 \\
\hline Appalachian Plateaus & 24 & -- & -- & -- & -- & -- & -- & -- & -- \\
\hline
\end{tabular}

Table 2. Statistical results from analysis of covariance of regressions relating bankfull-channel characteristics to drainage area in watersheds with and without carbonate bedrock in the Piedmont, Ridge and Valley, and Appalachian Plateaus Physiographic Provinces.

[N, number of stations; --, control]

\begin{tabular}{|c|c|c|c|c|c|c|c|c|c|}
\hline \multirow{3}{*}{$\begin{array}{c}\text { Physiographic } \\
\text { Province }\end{array}$} & \multirow{3}{*}{$\mathbf{N}$} & \multirow{2}{*}{\multicolumn{2}{|c|}{$\begin{array}{c}\begin{array}{c}\text { Bankfull cross-sectional } \\
\text { area }\end{array} \\
\text { p-value }\end{array}$}} & \multicolumn{2}{|c|}{ Bankfull discharge } & \multicolumn{2}{|c|}{ Bankfull width } & \multicolumn{2}{|c|}{ Bankfull mean depth } \\
\hline & & & & \multicolumn{2}{|c|}{ p-value } & \multicolumn{2}{|c|}{ p-value } & \multicolumn{2}{|c|}{ p-value } \\
\hline & & Intercept & Slope & Intercept & Slope & Intercept & Slope & Intercept & Slope \\
\hline Piedmont & 12 & 0.5492 & 0.2757 & 0.7605 & 0.3547 & 0.7155 & 0.6983 & 0.1173 & 0.0065 \\
\hline Ridge and Valley & 27 & .0904 & .8857 & .6142 & .0078 & .9265 & .0774 & .4265 & .9989 \\
\hline Appalachian Plateaus & 24 & -- & -- & -- & -- & -- & -- & -- & -- \\
\hline
\end{tabular}




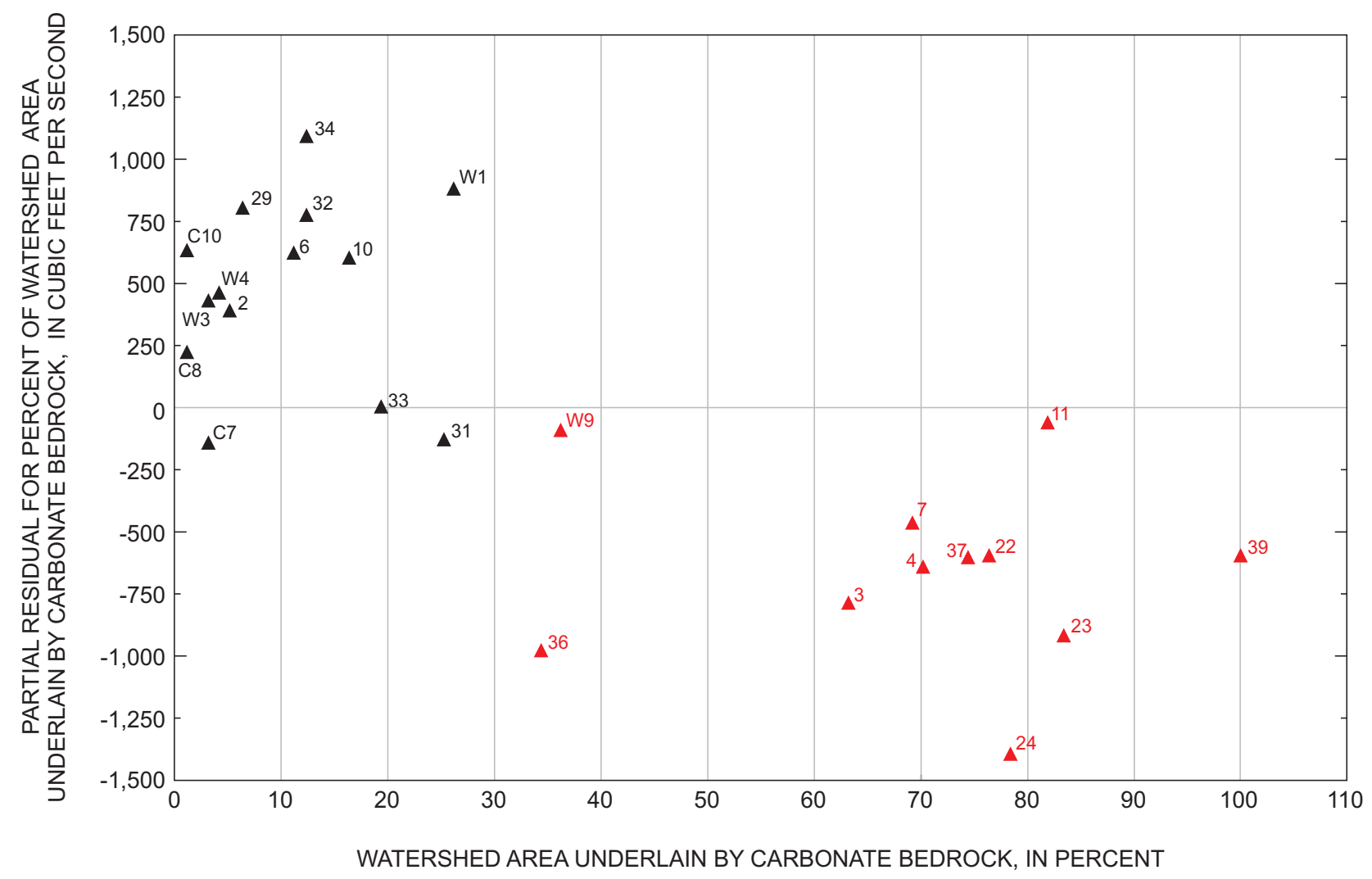

\section{EXPLANATION}
STATION IN WATERSHED UNDERLAIN BY
1 TO 30 PERCENT CARBONATE BEDROCK
14 STATIONS
STATION IN WATERSHED UNDERLAIN BY GREATER THAN 30 PERCENT CARBONATE BEDROCK
11 STATIONS

Figure 3. Relation between partial residuals for percent of watershed area underlain by carbonate bedrock and the percent of watershed area underlain by carbonate bedrock. See table 1 for information associated with cross-reference numbers identifying each station in this figure. 
The relation between bankfull discharge and drainage area developed from all 66 stations (fig. 4) is consistent with the pattern of partial residuals shown in figure 3. Stations in watersheds underlain by a significant percentage of carbonate bedrock generally have lower bankfull discharges compared to those having little or no carbonate bedrock (fig. 4). Evaluation of figures 3 and 4 leads to the conclusion that the effect of carbonate bedrock in this dataset can be characterized as either present or not; bankfull discharge in watersheds underlain by $\leq 30$ percent carbonate bedrock are influenced little and watersheds underlain by $>30$ percent carbonate bedrock are greatly influenced. Hence, stations were initially subset into those in watersheds underlain by $\leq 30$ percent carbonate bedrock and those in watersheds underlain by $>30$ percent carbonate bedrock.

ANCOVA was used to test for differences in the slope and intercept of regression lines relating bankfull discharge to drainage area in each subset. ANCOVA results indicate that the slope of the discharge regression developed from stations in watersheds underlain by $>30$ percent carbonate $(\mathrm{N}=11)$ is significantly less steep (p-value $=0.0020$ ) than the regression developed from stations in watersheds underlain by $\leq 30$ percent carbonate $(\mathrm{N}=55)$. The intercepts were not significantly different ( $\mathrm{p}$-value $=0.8377$ ).

On the basis of partial residuals from multiple regression (fig. 3), the relation between bankfull discharge and drainage area shown in figure 4, and confirmation through ANCOVA that treatment of carbonate bedrock as a class variable results in different regression lines, regional curves were developed to represent two settings independent of physiography: (1) a noncarbonate setting characterized by watersheds with carbonate bedrock underlying $\leq 30$ percent of the watershed area; and (2) a carbonate setting characterized by watersheds with carbonate bedrock underlying $>30$ percent of the watershed area.

Bankfull geometry and discharge data used to develop regional curves in the noncarbonate and carbonate settings are shown in tables 4 and 5, respectively. In general, regional curves developed with more data are less prone to violation of the underlying regression assumptions and have greater confidence than those developed with fewer data. Note that there are five times as many stations in noncarbonate setting $(\mathrm{N}=55)$ than in the carbonate setting $(\mathrm{N}=11)$. As a result, there is better representation of a range of drainage areas in the noncarbonate setting (table 4) thereby reducing the possibility of disproportionate influence by any one station. Regional curves developed from stations in carbonate settings are susceptible to influence from stations in watersheds less than $40 \mathrm{mi}^{2}$ because there are only two of them (table 5). The implications of differences between the noncarbonate and carbonate datasets are evaluated with statistical diagnostics that accompany the regional curves.

\section{Development and Evaluation of Regional Curves}

Regional curves are only estimates of the true relation between bankfull-response variables and drainage area because they are generated from a sample of stations intended to represent the population. The reliability of bankfull discharge and channel geometry estimated from regional-curve equations depends on how well the sample of stations represents the population, adherence to the assumptions of the underlying regression model, the fit of the curve to the data used to develop it, influence of any given data point on the slope of the curve, and the confidence in the curve over the range of represented drainage areas. Reliability of the regional curves and associated estimates of bankfull discharge and channel geometry are evaluated on the basis of statistical diagnostics included with the discussion of each set of regional curves. Computation of the diagnostics required transformation of the regional curves from commonly reported power functions (Cinotto, 2003; White, 2001; Dunne and Leopold, 1978) to log-linear functions. For the sole purpose of computing diagnostic statistics, each power function of the general form $y=a(D A)^{b}$ was transformed to the form:

$$
\log (y)=\log (a)+(b \times \log (D A))
$$

where

$\log$ is logarithm to base 10 ,

$\mathrm{y}$ is the bankfull-response variable,

a is the intercept of the regression line,

b is a coefficient of regression representing the slope of the regression line, and

DA is drainage area. 


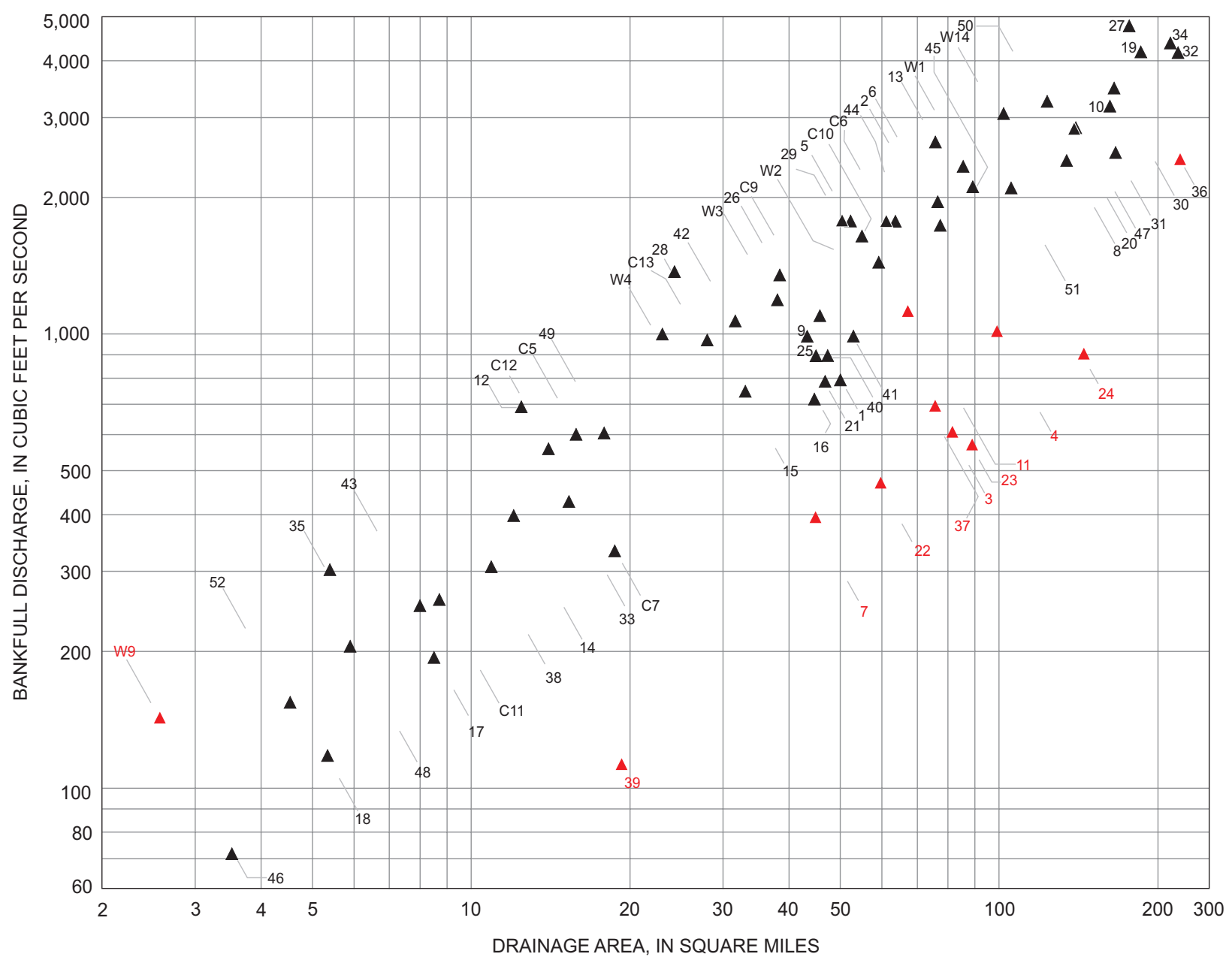

EXPLANATION

STATION IN WATERSHED UNDERLAIN BY LESS THAN
OR EQUAL TO 30 PERCENT CARBONATE BEDROCK

55 STATIONS

STATION IN WATERSHED UNDERLAIN BY GREATER

THAN 30 PERCENT CARBONATE BEDROCK 11 STATIONS

Figure 4. Relation between bankfull discharge and drainage area with emphasis on the influence of carbonate bedrock, Pennsylvania and selected areas of Maryland. See table 1 for information associated with cross-reference numbers identifying each station in this figure. 
Table 4. Channel characteristics from assessment of streamflow-gaging stations and associated stream reaches in noncarbonate settings of Pennsylvania and selected areas of Maryland.

$\left[\mathrm{mi}^{2}\right.$, square miles; WY, water year; $\mathrm{ft}$, feet; $\mathrm{ft}^{2}$, square feet; $\mathrm{ft}^{3} / \mathrm{s}$, cubic feet per second; $\mathrm{ft} / \mathrm{ft}$, feet per foot; mm, millimeter; D50, particle size larger than 50 percent of the cumulative sample; D84, particle size larger than 84 percent of the cumulative sample]

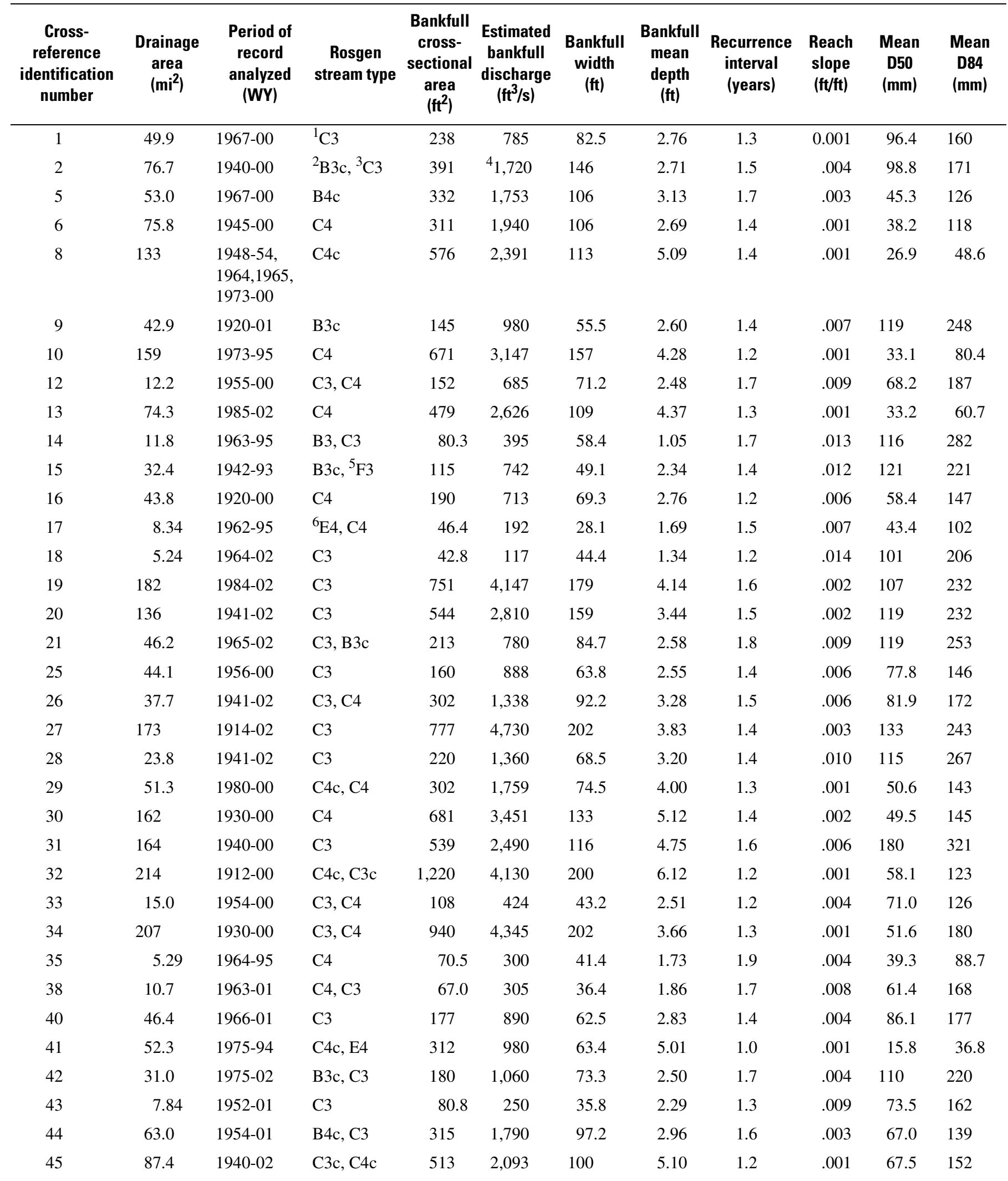


Table 4. Channel characteristics from assessment of streamflow-gaging stations and associated stream reaches in noncarbonate settings of Pennsylvania and selected areas of Maryland. — Continued

$\left[\mathrm{mi}^{2}\right.$, square miles; WY, water year; ft, feet; $\mathrm{ft}^{2}$, square feet; $\mathrm{ft}^{3} / \mathrm{s}$, cubic feet per second; $\mathrm{ft} / \mathrm{ft}$, feet per foot; $\mathrm{mm}$, millimeter; D50, particle size larger than 50 percent of the cumulative sample; D84, particle size larger than 84 percent of the cumulative sample]

\begin{tabular}{|c|c|c|c|c|c|c|c|c|c|c|c|}
\hline $\begin{array}{l}\text { Cross- } \\
\text { reference } \\
\text { identification } \\
\text { number }\end{array}$ & $\begin{array}{c}\text { Drainage } \\
\text { area } \\
\left(\mathbf{m i} \mathbf{i}^{2}\right)\end{array}$ & $\begin{array}{l}\text { Period of } \\
\text { record } \\
\text { analyzed } \\
\text { (WY) }\end{array}$ & $\begin{array}{l}\text { Rosgen } \\
\text { stream type }\end{array}$ & $\begin{array}{c}\text { Bankfull } \\
\text { cross- } \\
\text { sectional } \\
\text { area } \\
\left(\mathrm{ft}^{2}\right)\end{array}$ & $\begin{array}{c}\text { Estimated } \\
\text { bankfull } \\
\text { discharge } \\
\left(\mathrm{ft}^{3} / \mathrm{s}\right)\end{array}$ & $\begin{array}{c}\text { Bankfull } \\
\text { width } \\
\text { (ft) }\end{array}$ & $\begin{array}{c}\text { Bankfull } \\
\text { mean } \\
\text { depth } \\
\text { (ft) }\end{array}$ & $\begin{array}{l}\text { Recurrence } \\
\text { interval } \\
\text { (years) }\end{array}$ & $\begin{array}{l}\text { Reach } \\
\text { slope } \\
\text { (ft/ft) }\end{array}$ & $\begin{array}{c}\text { Mean } \\
\text { D50 } \\
(\mathrm{mm})\end{array}$ & $\begin{array}{c}\text { Mean } \\
\text { D84 } \\
(\mathrm{mm})\end{array}$ \\
\hline 46 & 3.45 & $\begin{array}{l}1985, \\
1988-97\end{array}$ & B3, C4 & 33.8 & 71.1 & 27.3 & 1.24 & 1.6 & 0.031 & 81.7 & 185 \\
\hline 47 & 137 & 1941-02 & $\mathrm{C} 4 \mathrm{c}, \mathrm{C} 4$ & 538 & 2,820 & 112 & 4.80 & 1.4 & .002 & 44.6 & 150 \\
\hline 48 & 5.78 & $1963-01$ & $\mathrm{E} 4, \mathrm{~B} 4 \mathrm{c}$ & 32.6 & 203 & 18.0 & 1.82 & 1.6 & .011 & 20.7 & 66.7 \\
\hline 49 & 17.5 & $1963-95$ & $\mathrm{C} 4 \mathrm{c}$ & 174 & 600 & 57.5 & 3.04 & 1.4 & .001 & 14.5 & 32.2 \\
\hline 50 & 121 & 1914-02 & B3c, F3 & 606 & 3,225 & 161 & 3.78 & 1.4 & .003 & 67.2 & 187 \\
\hline 51 & 104 & 1914-02 & $\mathrm{E} 4, \mathrm{C} 4$ & 409 & 2,080 & 72.5 & 5.65 & 1.5 & .002 & 52.3 & 175 \\
\hline 52 & 4.45 & 1987-02 & $\mathrm{F} 4, \mathrm{~F} 1$ & 24.1 & 153 & 29.1 & .84 & 1.2 & .012 & 41.0 & 93.3 \\
\hline${ }^{7} \mathrm{~W} 1$ & 85.5 & 1974-00 & $\mathrm{C} 4$ & 468 & 2,340 & 90.1 & 5.19 & 1.3 & .001 & 27.7 & 103 \\
\hline W2 & 59.1 & 1969-00 & $\mathrm{C} 3$ & 316 & 1,440 & 87.1 & 3.63 & 1.3 & .004 & 89.4 & 311 \\
\hline W3 & 38.0 & $1982-00$ & $\mathrm{C} 4$ & 304 & ${ }^{4} 1,190$ & 117 & 2.65 & 1.4 & .002 & 3.80 & 42.4 \\
\hline W4 & 23.0 & $1982-00$ & $\mathrm{C} 4$ & 202 & 1,000 & 96.5 & ${ }^{8}{ }_{2.09}$ & 1.5 & .004 & .80 & 70.4 \\
\hline${ }^{9} \mathrm{C} 5$ & 15.8 & 1970-01 & $\mathrm{C} 4, \mathrm{C} 5$ & 161 & 601 & 57.5 & 2.79 & 1.2 & .002 & 3.20 & 38.9 \\
\hline C6 & 61.1 & 1931-01 & $\mathrm{B} 4 \mathrm{c}$ & 303 & 1,772 & 69.6 & 4.35 & 1.2 & .002 & 48.1 & 153 \\
\hline C7 & 18.7 & $1960-01$ & $\mathrm{C} 4$ & 128 & 333 & 57.4 & 2.24 & 1.0 & .001 & 7.33 & 35.2 \\
\hline $\mathrm{C} 8$ & 45.8 & 1969-01 & $\mathrm{F} 4, \mathrm{~B} 4 \mathrm{c}$ & 179 & ${ }^{4} 1,097$ & 77.6 & 2.33 & 1.3 & .009 & 42.2 & 140 \\
\hline $\mathrm{C} 10$ & 55.0 & 1969-01 & $\mathrm{C} 4$ & 268 & 1,643 & 97 & 2.77 & 1.3 & .004 & 24.3 & 82.4 \\
\hline C11 & 8.7 & 1962-02 & $\mathrm{E} 4, \mathrm{C} 4$ & 63.7 & 260 & 29.2 & 2.22 & 1.3 & .004 & 43.6 & 146 \\
\hline $\mathrm{C} 12$ & 14.0 & 1982-01 & $\mathrm{B} 4 \mathrm{c}$ & 106 & 559 & 44.4 & 2.38 & 1.4 & .006 & 25.3 & 114 \\
\hline $\mathrm{C} 13$ & 28.0 & 1982-01 & $\mathrm{C} 4, \mathrm{~B} 4 \mathrm{c}$ & 189 & 970 & 68.2 & 2.77 & 1.4 & .004 & 27.0 & 78.9 \\
\hline W14 & 102 & 1948-00 & $\mathrm{C} 4$ & 616 & 3,060 & 101 & 6.12 & 1.5 & .001 & 22.4 & 95.8 \\
\hline
\end{tabular}

${ }^{1} \mathrm{C}$ type streams are characterized by Rosgen (1996) as single threaded channels with entrenchment ratios that are greater than $2.2 \mathrm{ft} / \mathrm{ft}$, width/depth ratios greater than $12 \mathrm{ft} / \mathrm{ft}$, sinuosity greater than $1.2 \mathrm{ft} / \mathrm{ft}$, and slopes ranging from less than $0.001-0.039 \mathrm{ft} / \mathrm{ft}$. Numbers and small letters following the "C" represent the bed material and a more refined slope category, respectively.

${ }^{2} \mathrm{~B}$ type streams are characterized by Rosgen (1996) as single threaded channels with moderate entrenchment ratios (1.4 - $\left.2.2 \mathrm{ft} / \mathrm{ft}\right)$, width/depth ratios greater than $12 \mathrm{ft} / \mathrm{ft}$, sinuosity greater than $1.2 \mathrm{ft} / \mathrm{ft}$, and slopes ranging from less than $0.020-0.099 \mathrm{ft} / \mathrm{ft}$. Numbers and small letters following the "B" represent the bed material and a more refined slope category, respectively.

${ }^{3}$ When two stream types are listed, the first is for the upstream-most cross section.

${ }^{4}$ Computed by slope-conveyance methods described in Rosgen (1996).

${ }^{5} \mathrm{~F}$ type streams are characterized by Rosgen (1996) as single threaded channels with entrenchment ratios that are less than $1.4 \mathrm{ft} / \mathrm{ft}$, width/depth ratios greater than $12 \mathrm{ft} / \mathrm{ft}$, sinuosity greater than $1.2 \mathrm{ft} / \mathrm{ft}$, and slopes ranging from less than $0.02-0.039 \mathrm{ft} / \mathrm{ft}$. Numbers following the "F" represent the bed material.

${ }^{6} \mathrm{E}$ type streams are characterized by Rosgen (1996) as single threaded channels with entrenchment ratios that are greater than $2.2 \mathrm{ft} / \mathrm{ft}$, width/depth ratios less than $12 \mathrm{ft} / \mathrm{ft}$, sinuosity greater than $1.5 \mathrm{ft} / \mathrm{ft}$, and slopes ranging from less than $0.02-0.039 \mathrm{ft} / \mathrm{ft}$. Numbers following the "E" represent the bed material.

${ }^{7}$ Cross-reference identification number preceded by a "W" indicates that data were originally published in White (2001).

${ }^{8}$ Previously reported as 1.94 in Cinotto (2003) and White (2001).

${ }^{9}$ Cross-reference identification number preceded by a "C" indicates that data were originally published in Cinotto (2003). 
Table 5. Channel characteristics from assessment of streamflow-gaging stations and associated stream reaches in carbonate settings of Pennsylvania and selected areas of Maryland.

$\left[\mathrm{mi}^{2}\right.$, square miles; WY, water year; $\mathrm{ft}$, feet; $\mathrm{ft}^{2}$, square feet; $\mathrm{ft}^{3} / \mathrm{s}$, cubic feet per second; ft/ft, feet per foot; mm, millimeter; --, no data; D50, particle size larger than 50 percent of the cumulative sample; D84, particle size larger than 84 percent of the cumulative sample]

\begin{tabular}{|c|c|c|c|c|c|c|c|c|c|c|c|}
\hline $\begin{array}{c}\text { Cross- } \\
\text { reference } \\
\text { identification } \\
\text { number }\end{array}$ & $\begin{array}{c}\text { Drainage } \\
\text { area } \\
\left(\mathrm{mi}^{2}\right)\end{array}$ & $\begin{array}{l}\text { Period of } \\
\text { record } \\
\text { analyzed } \\
\text { (WY) }\end{array}$ & $\begin{array}{l}\text { Rosgen } \\
\text { stream } \\
\text { type }\end{array}$ & $\begin{array}{l}\text { Bankfull } \\
\text { cross- } \\
\text { sectional } \\
\text { area } \\
\left(\mathrm{ft}^{2}\right)\end{array}$ & $\begin{array}{c}\text { Estimated } \\
\text { bankfull } \\
\text { discharge } \\
\left(\mathrm{ft}^{3} / \mathrm{s}\right)\end{array}$ & $\begin{array}{l}\text { Bankfull } \\
\text { width } \\
\text { (ft) }\end{array}$ & $\begin{array}{l}\text { Bankfull } \\
\text { mean } \\
\text { depth } \\
\text { (ft) }\end{array}$ & $\begin{array}{c}\text { Recurrence } \\
\text { interval } \\
\text { (years) }\end{array}$ & $\begin{array}{l}\text { Reach } \\
\text { slope } \\
(\mathrm{ft} / \mathrm{ft})\end{array}$ & $\begin{array}{c}\text { Mean } \\
\text { D50 } \\
(\mathrm{mm})\end{array}$ & $\begin{array}{c}\text { Mean } \\
\text { D84 } \\
(\mathrm{mm})\end{array}$ \\
\hline 3 & 80.8 & 1946-00 & ${ }^{1,2} \mathrm{C} 4 \mathrm{c}, \mathrm{C} 3 \mathrm{c}$ & 231 & 604 & 63.7 & 3.62 & 1.2 & 0.001 & 24.4 & 194 \\
\hline 4 & 98.2 & $1987-00$ & $\mathrm{C} 4$ & 215 & ${ }^{3} 1,006$ & 66.8 & 3.28 & 1.5 & .003 & 40.0 & 125 \\
\hline 11 & 66.5 & $1975-00$ & $\mathrm{C} 4$ & 305 & 1,114 & 111 & 2.96 & 1.4 & .001 & 24.2 & 76.3 \\
\hline 22 & 58.5 & $1985-00$ & $\mathrm{C} 4$ & 148 & 466 & 65.8 & 2.33 & 1.7 & .002 & 50.6 & 120 \\
\hline 23 & 87.2 & $1941-00$ & $\mathrm{C} 4, \mathrm{C} 3$ & 149 & 567 & 66.0 & 2.25 & 1.5 & .004 & 69.9 & 145 \\
\hline 24 & 142 & $1967-00$ & $\mathrm{C} 4 \mathrm{c}$ & 351 & 898 & 77.7 & 4.52 & 1.4 & .000 & 18.2 & 153 \\
\hline W9 & 2.57 & 1964-00 & $\mathrm{B} 5 \mathrm{c}$ & 24.0 & 143 & 17.8 & 1.36 & 1.2 & .009 & .25 & 55.7 \\
\hline
\end{tabular}

${ }^{1} \mathrm{C}$ type streams are characterized by Rosgen (1996) as single threaded channels with entrenchment ratios that are greater than $2.2 \mathrm{ft} / \mathrm{ft}$, width/depth ratios greater than $12 \mathrm{ft} / \mathrm{ft}$, sinuosity greater than $1.2 \mathrm{ft} / \mathrm{ft}$, and slopes ranging from less than $0.001-0.039 \mathrm{ft} / \mathrm{ft}$. Numbers and small letters following the "C" represent the bed material and a more refined slope category, respectively.

${ }^{2}$ When two stream types are listed, the first is for the upstream-most cross section.

${ }^{3}$ Computed by slope-conveyance methods described in Rosgen (1996).

${ }^{4} \mathrm{~B}$ type streams are characterized by Rosgen (1996) as single threaded channels with moderate entrenchment ratios $(1.4-2.2 \mathrm{ft} / \mathrm{ft})$, width/depth ratios greater than $12 \mathrm{ft} / \mathrm{ft}$, sinuosity greater than $1.2 \mathrm{ft} / \mathrm{ft}$, and slopes ranging from less than $0.020-0.099 \mathrm{ft} / \mathrm{ft}$. Numbers and small letters following the "B" represent the bed material and a more refined slope category, respectively.

${ }^{5}$ Bed substrate at station 39 (Marsh Run at Grimes, Md; 01617800) was carbonate bedrock

${ }^{6} \mathrm{E}$ type streams are characterized by Rosgen (1996) as single threaded channels with entrenchment ratios that are greater than $2.2 \mathrm{ft} / \mathrm{ft}$, width/depth ratios less than $12 \mathrm{ft} / \mathrm{ft}$, sinuosity greater than $1.5 \mathrm{ft} / \mathrm{ft}$, and slopes ranging from less than $0.02-0.039 \mathrm{ft} / \mathrm{ft}$. Numbers following the "E" represent the bed material.

\section{Regional Curves Developed for Noncarbonate Settings}

Regional curves representing the noncarbonate setting (figs. 5-8) have slopes that are significantly different than zero (p-values are less than 0.05; table 6) and normally distributed residuals ( $p$-values from Kolmogorov-Smirnov Goodness of Fit tests are greater than 0.05 ; table 6 ) that vary randomly with drainage area. Values of Cook's Distance, a measure of the influence by each station, indicate that no station has a disproportionate influence on any of the noncarbonate regional curves (table 6). Coefficient of determination $\left(\mathrm{R}^{2}\right)$ values indicate that 92 percent of variation in bankfull cross-sectional area and bankfull discharge is explained by drainage area compared to only 81 and 72 percent of the variation in bankfull width and mean depth, respectively (table 6). Wider 95-percent confi- dence intervals for the bankfull width (fig. 7) and mean depth (fig. 8) regional curves reflect greater uncertainty in estimates of regression parameters for these relations compared to the bankfull discharge (fig. 6) and cross-sectional area (fig. 5) relations. Thus, regional curves representing the noncarbonate setting are more reliable for estimation of bankfull discharge and cross-sectional area than bankfull width and mean depth.

ANCOVA results shown in tables 2 and 3 provide quantitative statistical evidence for combination of data across the Piedmont, Ridge and Valley, and Appalachian Plateaus Physiographic Provinces. Visual comparison of how bankfull characteristics vary among the physiographic provinces (figs. 5-8) provides additional qualitative support. In other words, it is difficult to visually determine if separate relations for each province would be different from one another given the variability of the data among the three provinces. 


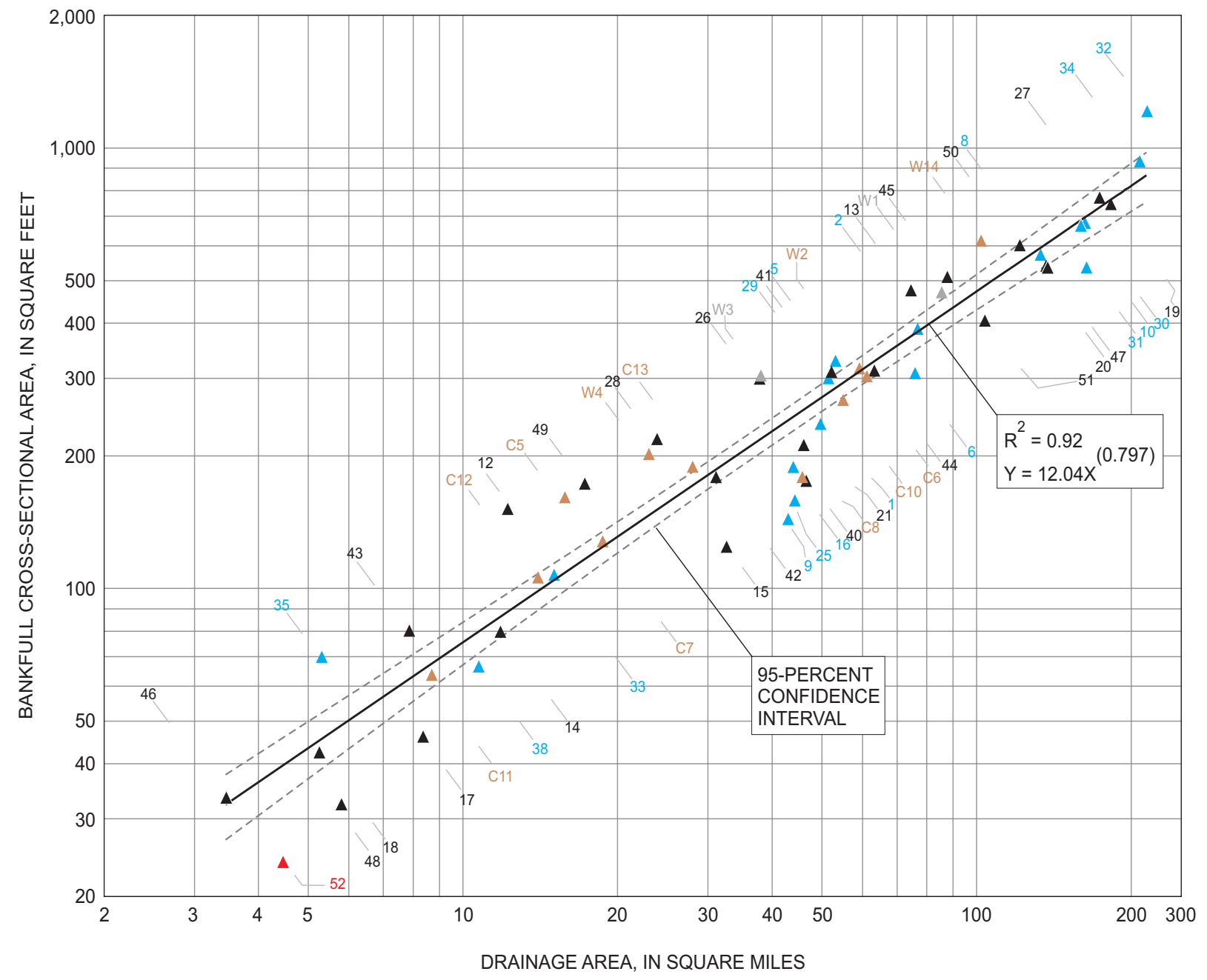

\section{EXPLANATION}

A STATION REPRESENTING THE PIEDMONT PHYSIOGRAPHIC PROVINCE

$\triangle \quad$ STATION REPRESENTING THE RIDGE AND VALLEY PHYSIOGRAPHIC PROVINCE

\ STATION REPRESENTING THE APPALACHIAN PLATEAUS PHYSIOGRAPHIC PROVINCE

$\triangle$ STATION REPRESENTING THE CENTRAL LOWLAND PHYSIOGRAPHIC PROVINCE

$\triangle \quad$ STATION REPRESENTING THE NEW ENGLAND PHYSIOGRAPHIC PROVINCE
11 STATIONS

17 STATIONS

24 STATIONS

1 STATION

2 STATIONS

Figure 5. Regional curve representing the relation between bankfull cross-sectional area and drainage area in noncarbonate settings of Pennsylvania and selected areas of Maryland. See table 1 for information associated with cross-reference numbers identifying each station in this figure. 


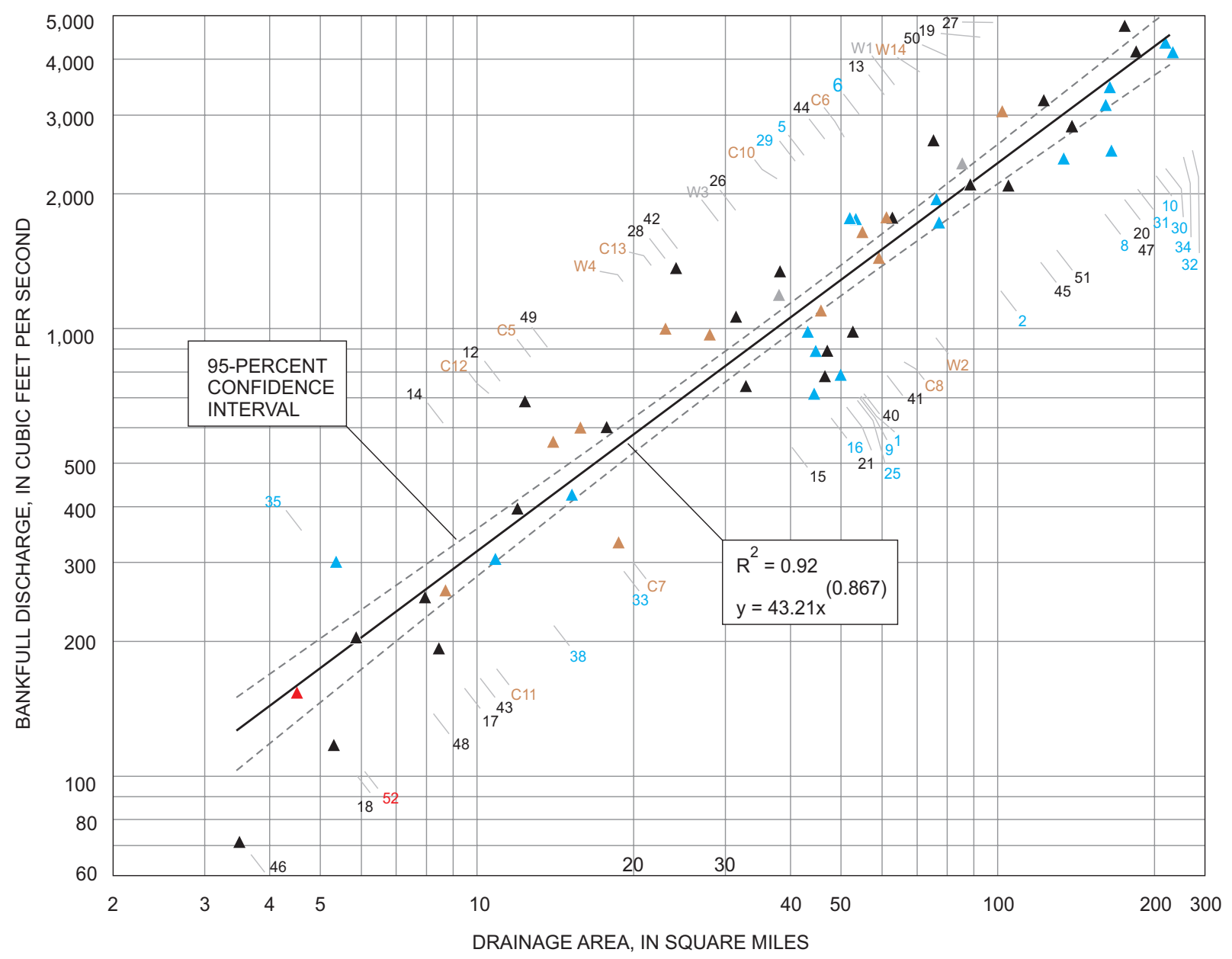

\section{EXPLANATION}

$\triangle \quad$ STATION REPRESENTING THE PIEDMONT PHYSIOGRAPHIC PROVINCE

$\triangle \quad$ STATION REPRESENTING THE RIDGE AND VALLEY PHYSIOGRAPHIC PROVINCE

\ STATION REPRESENTING THE APPALACHIAN PLATEAUS PHYSIOGRAPHIC PROVINCE

\ STATION REPRESENTING THE CENTRAL LOWLAND PHYSIOGRAPHIC PROVINCE

$\triangle$ STATION REPRESENTING THE NEW ENGLAND PHYSIOGRAPHIC PROVINCE
11 STATIONS

17 STATIONS

24 STATIONS

1 STATION

2 STATIONS

Figure 6. Regional curve representing the relation between bankfull discharge and drainage area in noncarbonate settings of Pennsylvania and selected areas of Maryland. See table 1 for information associated with cross-reference numbers identifying each station shown in this figure. 


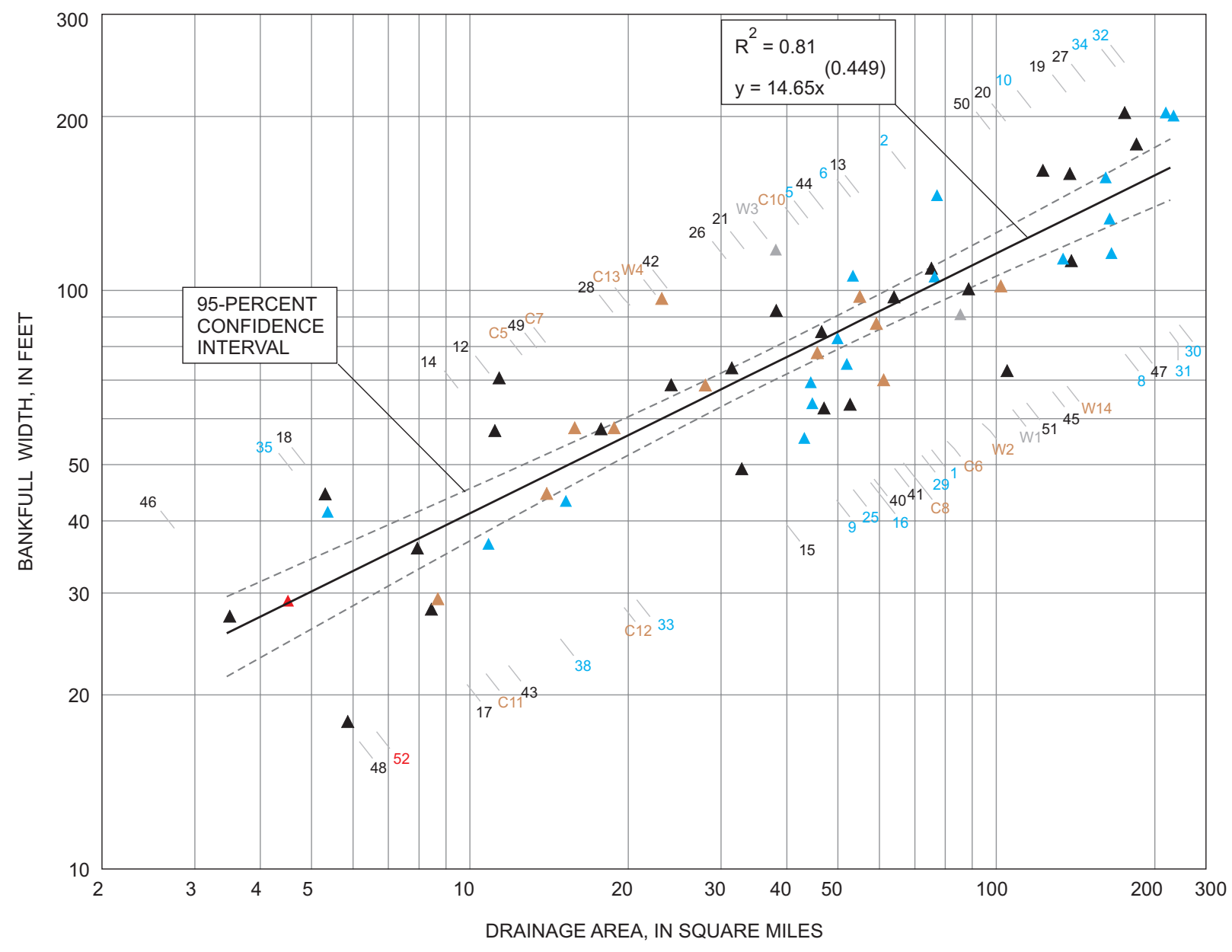

EXPLANATION

॥ STATION REPRESENTING THE PIEDMONT PHYSIOGRAPHIC PROVINCE

॥ STATION REPRESENTING THE RIDGE AND VALLEY PHYSIOGRAPHIC PROVINCE

- StATION REPRESENTING THE APPALACHIAN PLATEAUS PHYSIOGRAPHIC PROVINCE

॥ STATION REPRESENTING THE CENTRAL LOWLAND PHYSIOGRAPHIC PROVINCE

$\triangle$ STATION REPRESENTING THE NEW ENGLAND PHYSIOGRAPHIC PROVINCE
11 STATIONS

17 STATIONS

24 STATIONS

1 STATION

2 STATIONS

Figure 7. Regional curve representing the relation between bankfull width and drainage area in noncarbonate settings of Pennsylvania and selected areas of Maryland. See table 1 for information associated with cross-reference numbers identifying each station shown in this figure. 


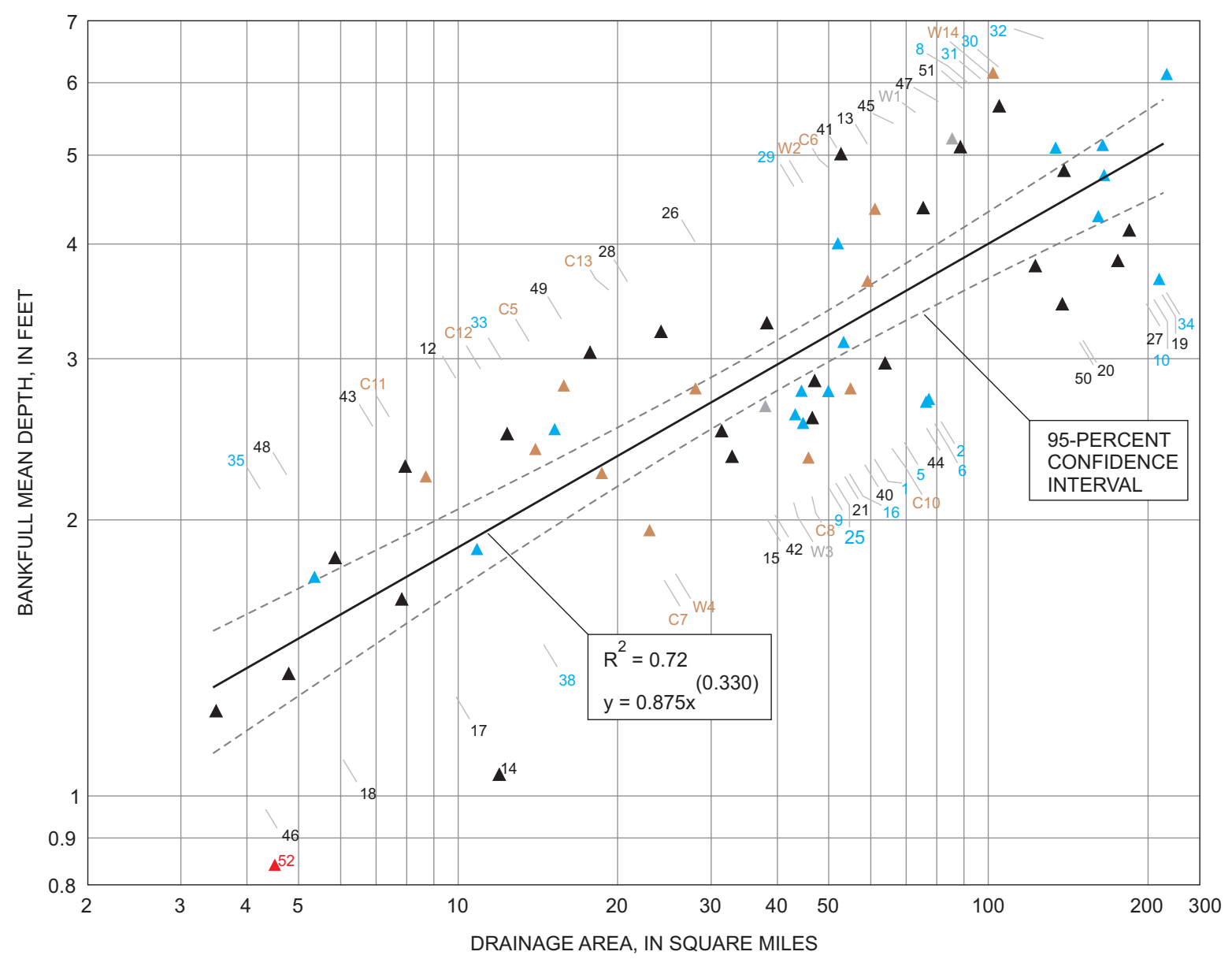

EXPLANATION

A STATION REPRESENTING THE PIEDMONT PHYSIOGRAPHIC PROVINCE

11 STATIONS

$\triangle$ STATION REPRESENTING THE RIDGE AND VALLEY PHYSIOGRAPHIC PROVINCE

17 STATIONS

- STATION REPRESENTING THE APPALACHIAN PLATEAUS PHYSIOGRAPHIC PROVINCE

24 STATIONS

$\triangle \quad$ STATION REPRESENTING THE CENTRAL LOWLAND PHYSIOGRAPHIC PROVINCE

1 STATION

$\triangle$ STATION REPRESENTING THE NEW ENGLAND PHYSIOGRAPHIC PROVINCE

2 STATIONS

Figure 8. Regional curve representing the relation between bankfull mean depth and drainage area in noncarbonate settings of Pennsylvania and selected areas of Maryland. See table 1 for information associated with cross-reference numbers identifying each station shown in this figure. 
Table 6. Coefficients and diagnostic statistics for regional curves relating bankfull characteristics to drainage area in noncarbonate settings of Pennsylvania and selected areas of Maryland.

[bf, bankfull; Q, discharge; N, number of observations; $\mathrm{R}^{2}$, coefficient of determination; Max, maximum; GOF, Kolmogorov-Smirnov Goodness of Fit Test]

\begin{tabular}{|c|c|c|c|c|c|c|c|c|c|}
\hline $\begin{array}{c}\text { Response } \\
\text { variable }\end{array}$ & $\begin{array}{l}\text { Intercept } \\
\text { (a) }\end{array}$ & $\begin{array}{l}\text { Slope } \\
\text { (b) }\end{array}$ & $\mathbf{N}$ & $\begin{array}{l}\begin{array}{c}p \text {-value } \\
\text { for }\end{array} \\
\text { regression } \\
\text { slope }^{1}\end{array}$ & $\begin{array}{l}\text { Residual } \\
\text { standard } \\
\text { error } \\
\text { (log units) }\end{array}$ & $\begin{array}{c}\text { Residual } \\
\text { standard } \\
\text { error } \\
\text { (percent) }\end{array}$ & $\mathrm{R}^{2}$ & $\begin{array}{c}\text { Cook's } \\
\text { Distance } \\
(\text { Max })^{3}\end{array}$ & $\begin{array}{c}\text { p-value } \\
\text { for } \\
\text { GOF }^{4}\end{array}$ \\
\hline bf area & 12.04 & 0.797 & 55 & $<0.0001$ & 0.11 & 26 & 0.92 & 0.20 & 0.9450 \\
\hline bf Q & 43.21 & .867 & 55 & $<.0001$ & .12 & 28 & .92 & .27 & .8818 \\
\hline bf depth & .875 & .330 & 55 & $<.0001$ & .10 & 23 & .72 & .28 & .8485 \\
\hline
\end{tabular}

\footnotetext{
${ }^{1} \mathrm{~A}$ p-value of less than or equal to 0.05 indicates that the slope of the regression line is different from zero.

${ }^{2}$ Residual standard error in percent determined from methods described by Gary Tasker, U.S. Geological Survey, written commun., 1978.

${ }^{3}$ Cook's distance is a measure of the influence that each streamflow-gaging station has on the regression relation. The maximum value of Cook's distance presented here corresponds to the station with the most influence. For the 55 watersheds representing the noncarbonate setting, a station is considered to have high influence if Cook's Distance is greater than 2.19.

${ }^{4}$ Kolmogorov-Smirnov Goodness of Fit tests the regression model assumption that residuals fit a normal distribution. This test is not a representation of how well the regression line fits the data. A p-value of greater than 0.05 indicates that regression residuals are normally distributed (Fail to reject the null hypothesis).
}

Additional data collected in similar physiographic settings outside of the study area will help determine if other regional curves are different from the ones presented here. New York State is presently (2005) developing regional curves for eight regions and has published curves for a portion of the Appalachian Plateaus Physiographic Province in the upper Susquehanna River Basin (Westergard and others, 2005). The regional curves presented here for noncarbonate settings of Pennsylvania are nearly identical to those in Westergard and others (2005).

\section{Regional Curves Developed for Carbonate Settings}

Regional curves representing the carbonate setting (figs. 9-12; table 7) generally are not as good as the corresponding noncarbonate curves (figs. 5-8; table 6). This is largely because drainage area does not explain variability caused by karst features, the dataset for the carbonate setting is statistically small $(\mathrm{N}=11$; table 7$)$, and watersheds less than $40 \mathrm{mi}^{2}$ are poorly represented. Unexplained variance and the small dataset results in less confidence (wider confidence intervals) for all carbonate regional curves (figs. 9-12) and higher residual standard errors for all but the bankfull depth regional curve (tables 6 and 7). Drainage area explains less variability in bankfull discharge and cross-sectional area of carbonate settings than for the noncarbonate setting $\left(\mathrm{R}^{2}\right.$ values are 0.88 and 0.73 compared to 0.92 and 0.92 , respectively; tables 7 and 6). Because only two gaged watersheds less than $40 \mathrm{mi}^{2}$ are available for regional-curve development in the carbonate setting, these relations are predisposed to disproportionate influence by the small watersheds. Values of Cook's Distance indicate that station W9, the smallest gaged watershed $\left(2.57 \mathrm{mi}^{2}\right.$; table 5), strongly influences the slope of the carbonate curves relating bankfull discharge, cross-sectional area, and mean depth to drainage area (table 7).

The implication of influence by station W9 was evaluated by comparing the regional curves presented in figures 9,10 , and 12 to corresponding regression equations developed without station W9. Exclusion of station W9 resulted in steeper positive slopes that intersect all the regional curves at a drainage area of approximately $84 \mathrm{mi}^{2}$. Hence, the regional curves developed for the carbonate setting may underestimate bankfull discharge, cross-sectional area, and mean depth, where drainage area is greater than $84 \mathrm{mi}^{2}$ and overestimate where drainage area is less than $84 \mathrm{mi}^{2}$. 


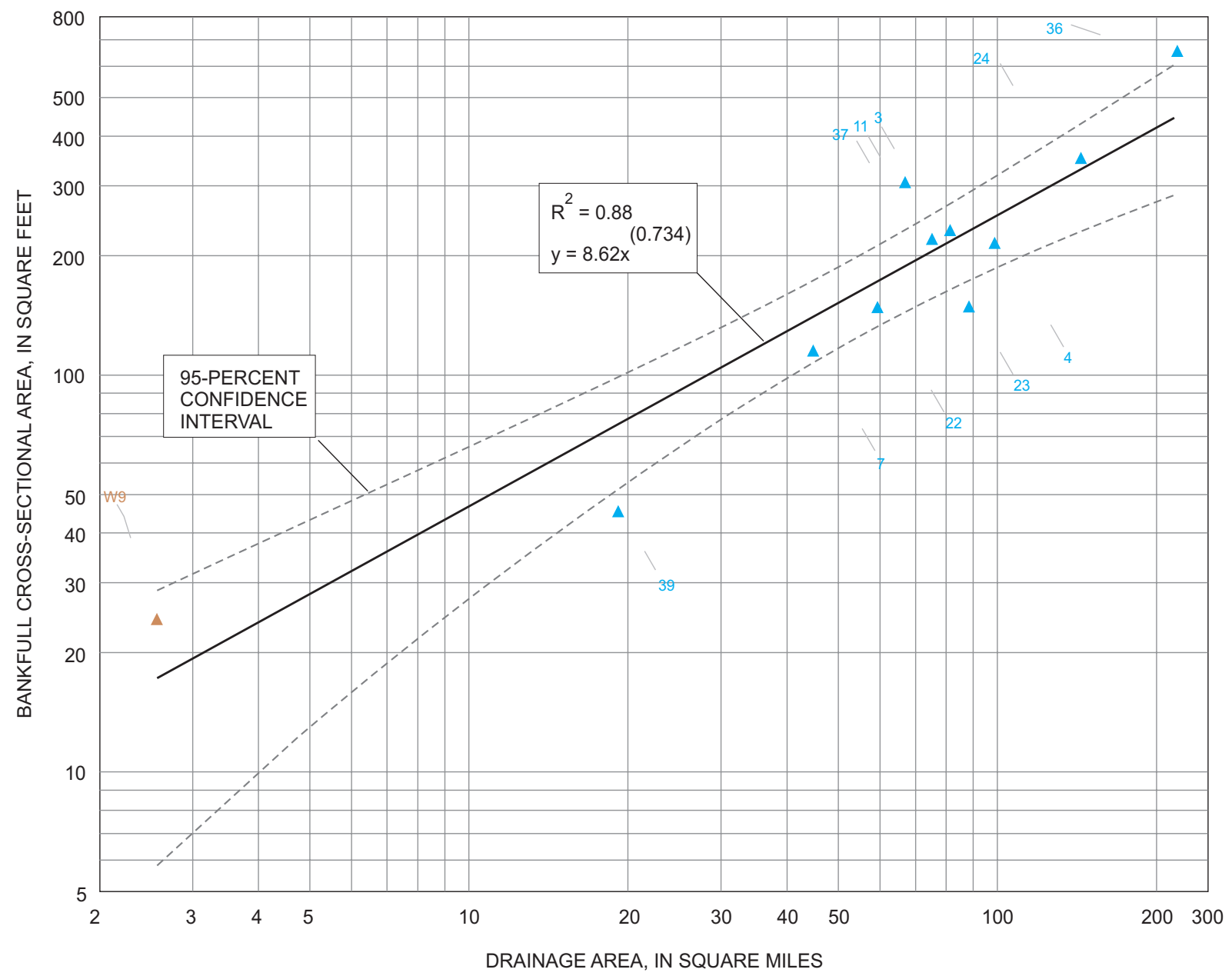

EXPLANATION

$\triangle \quad$ STATION REPRESENTING THE PIEDMONT PHYSIOGRAPHIC PROVINCE

1 STATION

- STATION REPRESENTING THE RIDGE AND VALLEY PHYSIOGRAPHIC PROVINCE

10 STATIONS

Figure 9. Regional curve representing the relation between bankfull cross-sectional area and drainage area for carbonate settings of Pennsylvania and selected areas of Maryland. See table 1 for information associated with crossreference numbers identifying each station shown in this figure. 


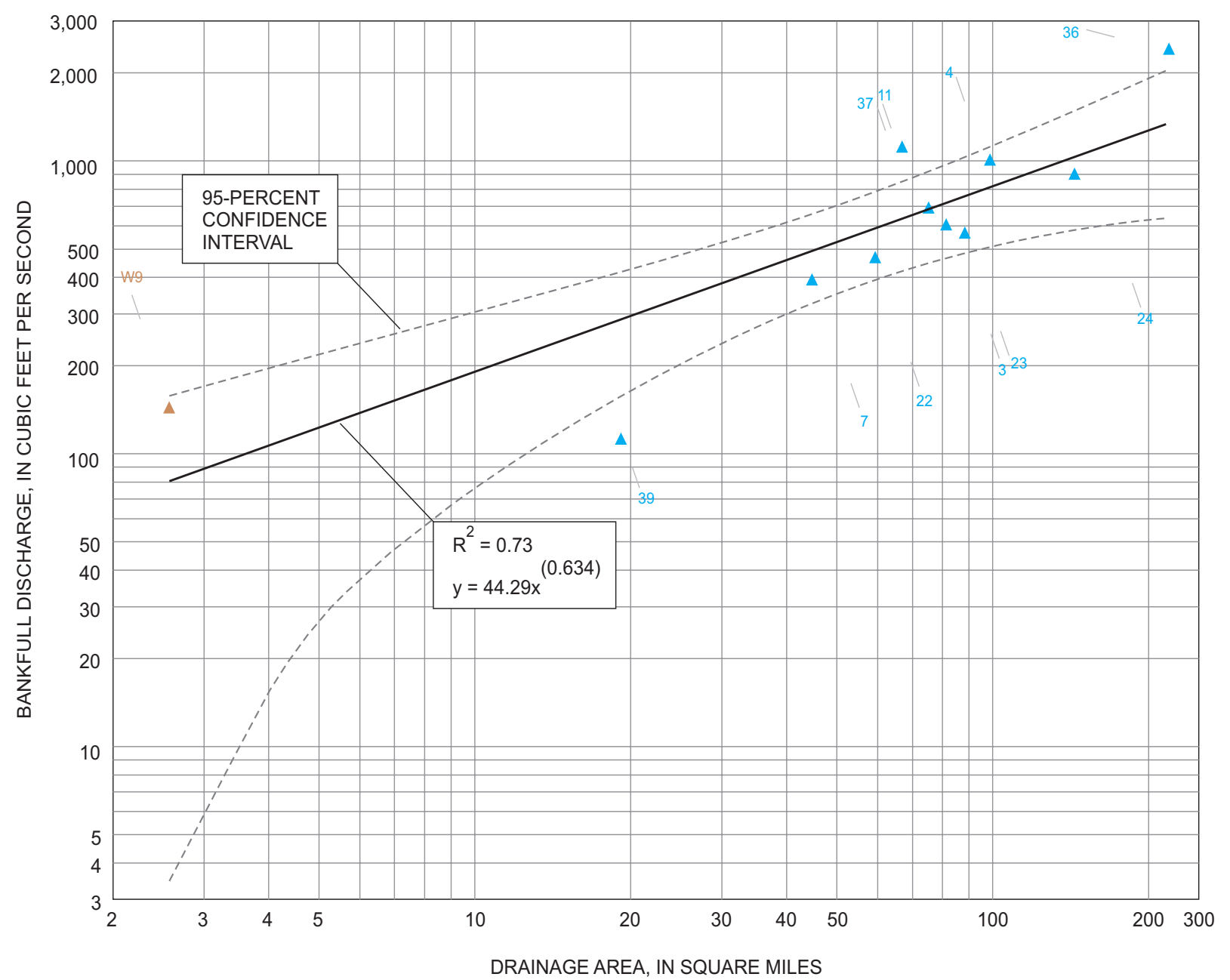

EXPLANATION

S STATION REPRESENTING THE PIEDMONT PHYSIOGRAPHIC PROVINCE

1 STATION

॥ STATION REPRESENTING THE RIDGE AND VALLEY PHYSIOGRAPHIC PROVINCE

10 STATIONS

Figure 10. Regional curve representing the relation between bankfull discharge and drainage area for carbonate settings of Pennsylvania and selected areas of Maryland. See table 1 for information associated with cross-reference numbers identifying each station shown in this figure. 


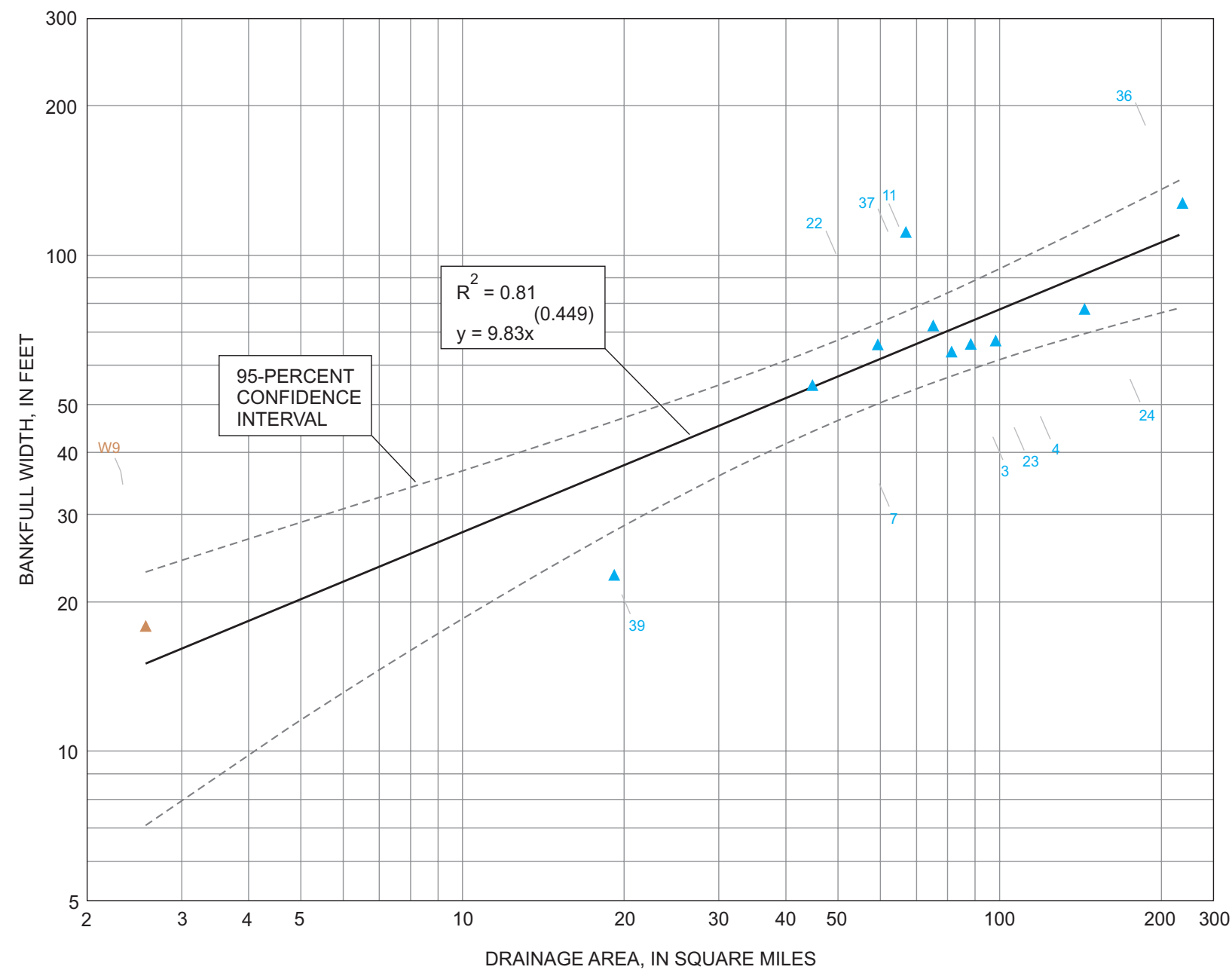

EXPLANATION

$\triangle \quad$ STATION REPRESENTING THE PIEDMONT PHYSIOGRAPHIC PROVINCE

1 STATION

$\triangle \quad$ STATION REPRESENTING THE RIDGE AND VALLEY PHYSIOGRAPHIC PROVINCE

10 STATIONS

Figure 11. Regional curve representing the relation between bankfull width and drainage area for carbonate settings of Pennsylvania and selected areas of Maryland. See table 1 for information associated with cross-reference numbers identifying each station shown in this figure. 


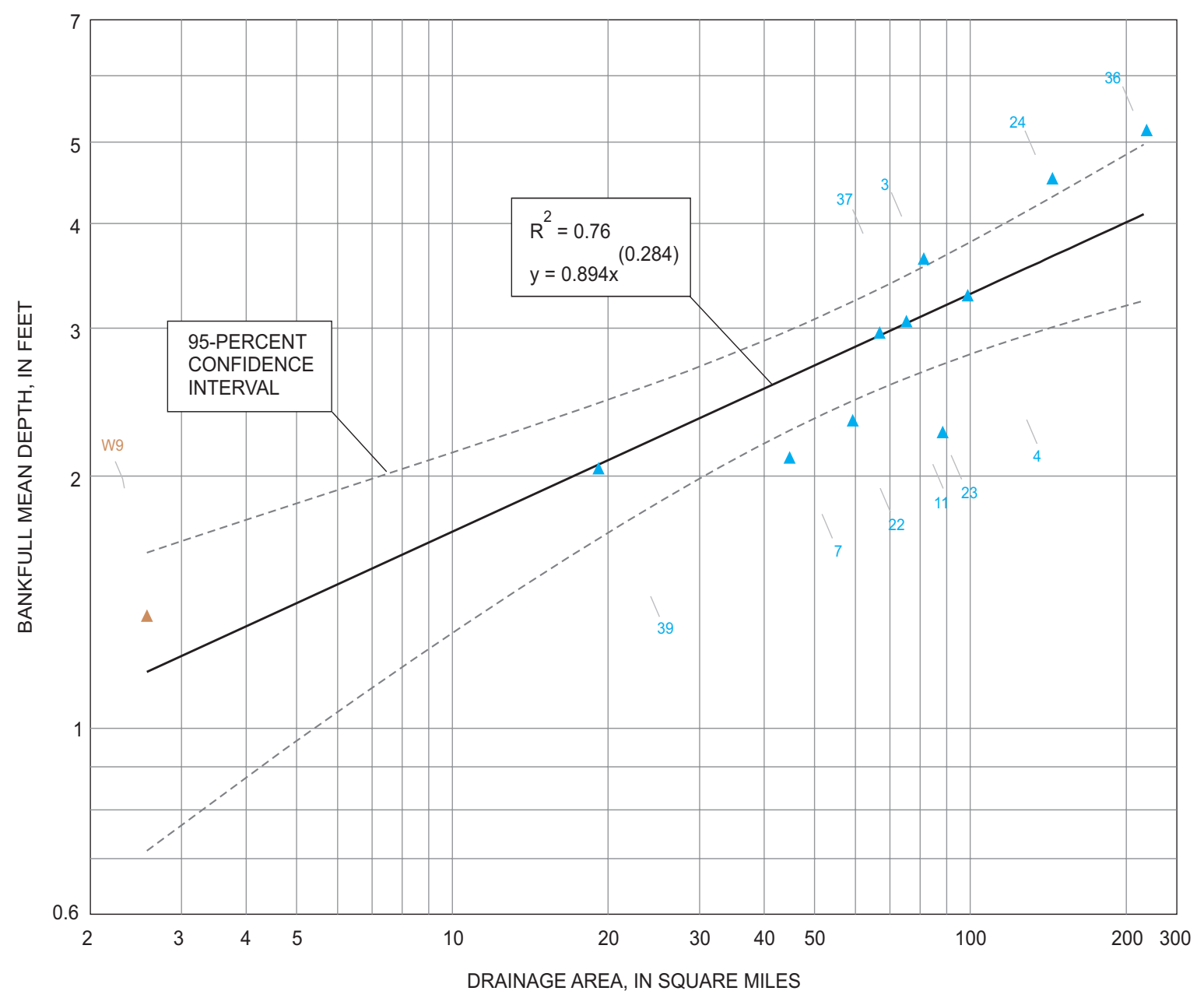

EXPLANATION

$\triangle$ STATION REPRESENTING THE PIEDMONT PHYSIOGRAPHIC PROVINCE

1 STATION

॥ STATION REPRESENTING THE RIDGE AND VALLEY PHYSIOGRAPHIC PROVINCE

10 STATIONS

Figure 12. Regional curve representing the relation between bankfull mean depth and drainage area for carbonate settings of Pennsylvania and selected areas of Maryland. See table 1 for information associated with cross-reference numbers identifying each station shown in this figure. 
Table 7. Coefficients and diagnostic statistics for regional curves relating bankfull characteristics to drainage area in carbonate settings of Pennsylvania and selected areas of Maryland.

[bf, bankfull; Q, discharge; N, number of observations; $\mathrm{R}^{2}$, coefficient of determination; Max, maximum; GOF, Kolmogorov-Smirnov Goodness of Fit Test]

\begin{tabular}{|c|c|c|c|c|c|c|c|c|c|}
\hline $\begin{array}{l}\text { Response } \\
\text { variable }\end{array}$ & $\begin{array}{l}\text { Intercept } \\
\text { (a) }\end{array}$ & $\begin{array}{l}\text { Slope } \\
\text { (b) }\end{array}$ & $\mathbf{N}$ & $\begin{array}{l}\text { p-value } \\
\text { for } \\
\text { regression } \\
\text { slope }^{1}\end{array}$ & $\begin{array}{c}\text { Residual } \\
\text { standard } \\
\text { error } \\
\text { (log units) }\end{array}$ & $\begin{array}{c}\text { Residual } \\
\text { standard } \\
\text { error } \\
\text { (percent) })^{2}\end{array}$ & $\mathbf{R}^{2}$ & $\begin{array}{c}\text { Cook's } \\
\text { Distance } \\
(\text { Max })^{3}\end{array}$ & $\begin{array}{c}p \text {-value } \\
\text { for } \\
\text { GOF }^{4}\end{array}$ \\
\hline bf area & 8.62 & 0.734 & 11 & $<0.0001$ & 0.15 & 36 & 0.88 & 7.8 & 0.9787 \\
\hline bf Q & 44.29 & .634 & 11 & .0009 & .21 & 51 & .73 & 8.4 & .8045 \\
\hline bf depth & .894 & .284 & 11 & .0004 & .09 & 21 & .76 & 3.6 & .6392 \\
\hline
\end{tabular}

\footnotetext{
${ }^{1} \mathrm{~A}$ p-value of less than or equal to 0.05 indicates that the slope of the regression line is different from zero.

${ }^{2}$ Residual standard error in percent determined from methods described by Gary Tasker, U.S. Geological Survey, written commun., 1978.

${ }^{3}$ Cook's distance is a measure of the influence that each streamflow-gaging station has on the regression relation. The maximum value of Cook's distance presented here corresponds to the station with the most influence. For the 11 watersheds representing the carbonate setting, a station is considered to have high influence if Cook's Distance is greater than 2.81 .

${ }^{4}$ Kolmogorov-Smirnov Goodness of Fit tests the regression model assumption that residuals fit a normal distribution. This test is not a representation of how well the regression line fits the data. A p-value of greater than 0.05 indicates that regression residuals are normally distributed (Fail to reject the null hypothesis).
}

\section{Limitations}

The limitations of this investigation fall into two categories: (1) limitations related to the selection of streamflow-gaging stations and the associated bankfull channel, and (2) the development of regional curves. These limitations and the associated constraints on application of regional curves presented in this report are discussed below.

\section{Limitations Related to Selection of Streamflow-Gaging Stations and the Bankfull Channel}

Urbanization generally increases the size and frequency of floods because of impervious surfaces that convey runoff to streams much faster than natural surfaces that provide for infiltration. In response, stream channels commonly widen or downcut to increase their cross-sectional area so higher, more frequent flows can be accommodated (Doll and others, 2002). The filtering criterion of approximately 20 percent urban land use used in this study is a surrogate for impervious cover and is intended to exclude watersheds that may introduce unwanted variability in bankfull characteristics. Note that not all urban land use is impervious, and therefore the percent of urban land use overestimates the percent of impervious cover. Schueler (1995) linked watersheds with impervious cover as low as 10 percent to instability and erosion of channels. Thus, if the urban filtering criterion failed to exclude some watersheds with greater than 10 percent impervious cover, unwanted variability may have been introduced.

With the exception of one station, the assumption used in this investigation is that the channel-forming bankfull discharge is within the 1- to 2-year recurrence range. The literature contains debate about the validity of this assumption. Thorne and others (1997) state, "The widely reported assertion that bankfull discharge occurs on average once every 1 to 2 years is now seen as an oversimplification." If runoff patterns at a streamflowgaging station were such that the bankfull discharge occurs more, or less, often than this 1- to 2-year frequency, the recurrence-interval criterion did not substantiate identification of the bankfull channel.

The stability of the reaches included in this study-in terms of sediment transport, hydraulic conveyance, and degradation and (or) aggradation of the stream reaches-has not been confirmed, and long-term monitoring of the reaches has not been completed. Stream channels in transitional states may have introduced increased variability into the regional curves. Hence, regional curves are best used to initially confirm or refute field identification of the bankfull channel and are not intended as the sole tool to develop design criteria for stream restoration. 


\section{Limitations Related to Development of Regional Curves}

Bankfull channel geometry and discharge data are inherently variable. Regional curves use drainage area to explain this variability and are described by a best-fit equation that can be used to estimate bankfull characteristics. The quality of these estimates depends on how well the population is represented (number of stations available) and variability caused by factors other than drainage area. Regional curves developed for the noncarbonate setting are statistically more robust than those for the carbonate setting because there were more stations available (55 compared to 11) and the variability resulting from carbonate bedrock was minimized. Note, however, that very few values of bankfull characteristics determined from field data collected in the noncarbonate setting fall directly on any of the regional curves. This serves to emphasize that field verification is important for application of regional curves in any setting.

The small number of stations in carbonate settings and the variability introduced by carbonate bedrock are the primary limitations of the regional curves developed for carbonate settings. These relations are disproportionately influenced by the smallest watershed selected for this study and have poor confidence, particularly below a drainage area of $40 \mathrm{mi}^{2}$. Some stations in the carbonate setting, including W9 (Sucker Run near Coatesville, $\mathrm{Pa}$; table 1), may relate better to noncarbonate settings. Because of inherent variability in the distribution of karst features and their effect on streamflow (Kochanov and Reese, 2003), some stations may be influenced by carbonate bedrock even if the percentage underlying the watershed is 30 percent or less. Additional bankfull discharge and channel-geometry data from small watersheds in carbonate settings might reduce the influence of small watersheds, increase confidence, and generally improve the carbonate regional curves.

\section{Constraints on Application of Regional Curves}

The limitations presented above result in some constraints for the application of regional curves presented in this report. These constraints are briefly described below:

1. Regional curves in this report apply only to streams within the study area draining watersheds no larger or smaller than the range of watershed areas used to develop the curves.

2. The regional curves apply to streams in watersheds having land use and streamflow that is consistent with the criteria used for station selection.

3. None of the regional curves are intended as the sole tool for estimation of bankfull characteristics but may supplement field identification of the bankfull channel when used in conjunction with field-verified bankfull indicators, streamflow-frequency analysis, or other supporting evidence.
4. Application of regional curves developed for the carbonate setting may require additional rigor in field verification of the bankfull channel because of unexplained variability from karst features.

\section{Summary and Conclusions}

The U.S. Geological Survey, in cooperation with the Pennsylvania Department of Environmental Protection, Canaan Valley Institute, and Susquehanna River Basin Commission conducted a study between December 1999 and December 2003 to develop regional curves relating bankfull-channel geometry and discharge to drainage area. This information can be used by water-resources managers and others to support stream-restoration efforts across Pennsylvania and northern Maryland. The initial field work began in the Piedmont Physiographic Province and continued westward across the Ridge and Valley and Appalachian Plateaus Physiographic Provinces. Data from 14 streamflow-gaging stations previously used to develop regional curves in the Piedmont were combined with data collected at 52 additional stations within watersheds in the Ridge and Valley and Appalachian Plateaus Physiographic Provinces.

Analysis of Covariance (ANCOVA) and multiple regression results indicate that the percentage of carbonate bedrock underlying a watershed explains more variability in bankfull characteristics than physiography. Consequently, there was little evidence to support development of separate regional curves for the Ridge and Valley, Piedmont, and Appalachian Plateaus Physiographic Provinces. Instead, more statistical power was achieved by combining bankfull-channel geometry and discharge data across physiographic provinces to develop regional curves for two settings: (1) a noncarbonate setting characterized by watersheds with carbonate bedrock underlying less than or equal to 30 percent of the watershed area, and (2) a carbonate setting characterized by watersheds with carbonate bedrock underlying greater than 30 percent of the watershed area.

The reliability of bankfull discharge and channel geometry estimated from regional-curve equations depends on how well the sample of stations represents the population, adherence to the assumptions of the underlying regression model, the fit of the regional curve to the data used to develop it, the influence of a given data point on the slope of the curves, and the confidence in the curve over the range of represented drainage areas. In general, the regional curves representative of the noncarbonate setting are more robust than those for the carbonate setting because there were more stations available and drainage area explains more variability in the noncarbonate setting. Of the noncarbonate regional curves, drainage area explains the most variability in bankfull cross-sectional area and bankfull discharge $\left(\mathrm{R}^{2}\right.$ values $\left.=0.92\right)$. Less variability is explained in bankfull width and depth $\left(\mathrm{R}^{2}=0.81\right.$ and 0.72 , respectively).

Regional curves for carbonate settings were developed with fewer stations than the noncarbonate regional curves and are characterized by greater unexplained variance, less confi- 
dence, lower $\mathrm{R}^{2}$ values, and higher residual standard errors. Poor representation of watersheds less than $40 \mathrm{mi}^{2}$ causes carbonate regional curves for bankfull cross-sectional area, bankfull discharge, and bankfull depth to be disproportionately influenced by station W9 (Sucker Run near Coatesville, Pa.), which is in the smallest watershed selected for this investigation $\left(2.57 \mathrm{mi}^{2}\right)$. Consequently, the applicability of the carbonate regional curves may depend on how well Sucker Run represents the carbonate setting. Additional bankfull discharge and channel-geometry data from small watersheds might reduce the influence of Sucker Run, increase confidence, and generally improve regional curves representing the carbonate setting.

Limitations associated with streamflow-gaging station selection and development of the curves constrain the application of regional curves presented in this report. Regional curves presented here apply only to streams within the study area that drain watershed having land use, streamflow regulation, and drainage areas that are consistent with the criteria used for station selection. Use of regional curves developed for the carbonate setting may require additional rigor in field verification of the bankfull channel because of unexplained variability from karst features. Regardless of the setting, the regional curves presented here are not intended as the sole tool for estimation of bankfull characteristics. Instead, they may supplement field identification of the bankfull channel when used in conjunction with field-verified bankfull indicators, streamflow frequency analysis, or other supporting evidence.

\section{Acknowledgments}

Many parties supported this investigation. Special thanks is due to the Pennsylvania Department of Environmental Protection (PaDEP) for providing support through Section 319 of the Federal Clean Water Act administered by the U.S. Environmental Protection Agency. Additional support also was provided by the Canaan Valley Institute and the Susquehanna River Basin Commission. Recognition is due to Carl DeLuca and Tom Dillingham of PaDEP for taking time out of their schedules to help collect data from several streams in the Ridge and Valley Physiographic Province. Donald Williams along with Scott Sorber of USGS collected and compiled the majority of field data in the Appalachian Plateaus Physiographic Province. Scott Sorber also provided a tremendous amount of support for data collection in the Ridge and Valley Physiographic Province. Kirk White and Peter Cinotto of USGS originally published the data from the Piedmont and New England Physiographic Provinces used in this report (White, 2001, and Cinotto, 2003, respectively) and provided invaluable support for field data collection and data analysis throughout the investigation. Advice on selection of statistical procedures and interpretation of data analyses was provided by Edward Koerkle of USGS. This report improved greatly from technical reviews by PaDEP, Bradford County Conservation District, and USGS.

\section{References Cited}

Cinotto, P.J., 2003, Development of regional curves of bankfull-channel geometry and discharge for streams in the nonurban Piedmont Physiographic Province, Pennsylvania and Maryland: U.S. Geological Survey Water-Resources Investigation Report 03-4014, 27 p.

Cuff, D.J., Young W.J., Muller, E.K., Zelinsky, W., and Abler, R.F., eds., 1989, The atlas of Pennsylvania: Philadelphia, Temple University Press, p. 18-25.

Doll, B.A., Wise-Frederick, D.E., Buckner, C.M., Wilkerson, S.D., Harman, W.A., Smith, R.E., and Spooner, J., 2002, Hydraulic geometry relationships for urban streams throughout the Piedmont of North Carolina: Journal of the American Water Resources Association, v. 38., p. 641-651.

Dunne, T., and Leopold, L.B., 1978, Water in environmental planning: San Francisco, W.H. Freeman, 818 p.

Helsel, D.R., and Hirsch, R.M., 2002, Statistical methods in water resources: U.S. Geological Survey Techniques of Water-Resources Investigations, book 4, chap. A3, p. 142-147.

Kochanov, W.E., and Reese, S.O., 2003, Density of mapped karst features in south-central and southeastern Pennsylvania [online]: Pennsylvania Geologic Survey, map 68, scale 1:300,000, accessed June 24, 2004, at URL http://www.dcnr.state.pa.us/topogeo/map68/downloads/map68.pdf.

Langland, M.J., Lietman, P.L., Hoffman, S.A., 1995, Synthesis of nutrient and sediment data for watersheds within the Chesapeake Bay drainage basin: U.S. Geological Survey Water-Resources Investigations Report 95-4233, 46 p. plus appendixes.

Leopold, L.B., 1994, A view of the river: Cambridge, Mass., Harvard University Press, 298 p.

Lumia, R., 1991, Regionalization of flood discharges for rural, unregulated streams in New York, excluding Long Island: U.S. Geological Survey Water-Resources Investigations Report 90-4197, 119 p.

McCandless, T.L., and Everett, R.A., 2002, Maryland stream survey-Bankfull discharge and channel characteristics of streams in the Piedmont Hydrologic Region: U.S. Fish and Wildlife Service Chesapeake Bay Field Office, CBFO-S02-01, $40 \mathrm{p}$.

McCandless, T.L., 2003a, Maryland stream survey—Bankfull discharge and channel characteristics of streams in the Allegheny Plateau and the Valley and Ridge Hydrologic Regions: U.S. Fish and Wildlife Service Chesapeake Bay Field Office, CBFO-S03-01, 33 p.

McCandless, T.L., 2003b, Maryland stream survey—Bankfull discharge and channel characteristics of streams in the Coastal Plain Hydrologic Region: U.S. Fish and Wildlife Service Chesapeake Bay Field Office, CBFO-S03-02, 29 p. 
Pennsylvania Department of Conservation and Natural Resources, comp. 1995, Physiographic Provinces (Version 1) [online]: Bureau of Topographic and Geologic Survey, map 13, accessed July 29, 2004, at URL http:// www.pasda.psu.edu/documents.cgi/dcnr/pages/ pags_physprov5k.xml.

Pennsylvania Department of Conservation and Natural Resources, 2000a, Glacial deposits of Pennsylvania (2d ed.) [online]: Bureau of Topographic and Geologic Survey, map 59, accessed November 2004, at URL http:// www.dcnr.state.pa.us/topogeo/maps/map59.pdf.

Pennsylvania Department of Conservation and Natural Resources, 2000b, Limestone and dolomite distribution in Pennsylvania (4th ed.) [online]: Bureau of Topographic and Geologic Survey, map 15, accessed November 2004, at URL http://www.dcnr.state.pa.us/topogeo/maps/map15.pdf.

Rosgen, D., 1996, Applied river morphology: Minneapolis, Minn., Printed Media Companies, 352 p.

SAS Institute, Inc., 1990, SAS procedures guide (3d. ed.): Cary, N.C., SAS Institute, Inc., version 6, 705 p.

Schueler, T., 1995, The importance of imperviousness: Watershed Protection Techniques, v. 1, p. 100-111.

Sevon, W.D., comp., 2000, Physiographic provinces of Pennsylvania (4th ed.) [online]: Bureau of Topographic and Geologic Survey map 13, accessed July 28, 2004, at URL http://www.dcnr.state.pa.us/topogeo/maps/map13.pdf.

Stuckey, M.H., and Reed, L.A., 2000, Techniques for estimating magnitude and frequency of peak flows for Pennsylvania streams: U.S. Geological Survey Water-Resources Investigations Report 00-4189, 20 p. plus appendixes.

Thorne, C.R., Hey, R.D., and Newson, M.D., 1997, Applied fluvial geomorphology for river engineering and management: Chichester, England, John Wiley and Sons, 376 p.
U.S. Geological Survey, 1996, National land cover data [online]: U.S. Geological Survey, accessed May 17, 2001, at URL http://edcwww.cr.usgs.gov/programs/lccp/nationallandcover.html.

U.S. Geological Survey, 2003, User's manual for the national water information system of the U.S. Geological Survey-Automated data processing system (ADAPS) [online]: U.S. Geological Survey Open-File Report 03-89, version 4.2, accessed July 28, 2004, at URL http://wwwnwis.er.usgs.gov/nwisdocs4_2/adaps/adapscover.pdf.

Water Resources Council, Hydrology Committee, 1981, Guidelines for determining flood flow frequencies: Washington, D.C., Bulletin 17B, variously paged.

Westergard, B.E., Mulvihill, C.I., Ernst, A.G., and Baldigo, B.P., 2005, Regionalized equations for bankfull-discharge and channel characteristics of streams in New York State-Hydrologic region 5 in central New York: U.S. Geological Survey Scientific Investigations Report 2004-5247, $16 \mathrm{p}$.

White, E.L., 1976, Role of carbonate rocks in modifying flood flow behavior: Water Resources Bulletin, v. 12, no. 2, p. 351-369.

White, K.E., 2001, Regional curve development and selection of a reference reach in the non-urban, lowland sections of the Piedmont Physiographic Province, Pennsylvania and Maryland: U.S. Geological Survey Water-Resources Investigation Report 01-4146, 20 p.

Wolman, M.G., and Miller, J.P., 1960, Magnitude and frequency of forces in geomorphic processes: Journal of Geology, v. 68, p. 54-74. 


\section{Glossary}

The terms in this glossary were compiled from numerous sources. Some definitions have been modified for use within this report.

bankfull channel The active stream channel during the bankfull discharge.

bankfull cross-sectional area The cross-sectional area of the bankfull channel measured perpendicular to the streamflow.

bankfull discharge The most effective streamflow for moving sediment, forming or removing bars, forming or changing bends and meanders, and generally doing work that results in the average morphological characteristics of channels (Dunne and Leopold, 1978).

bankfull mean depth The mean depth of the bankfull channel measured perpendicular to the streamflow.

bankfull width The width of the bankfull channel measured perpendicular to the streamflow.

coefficient of determination $\left(\mathbf{R}^{2}\right)$ Measures the amount of variation in the bankfull response variable (bankfull cross-sectional area, discharge, width, or depth) that is accounted for by the variation in the explanatory variable (drainage area) (Helsel and Hirsch, 2002).

Cook's Distance Measures the influence of each observation on the regional curves. An observation is considered to have high influence if the test statistic " $\mathrm{D}_{i}$ " is greater than the F value corresponding to the tenth percentile of the F distribution. " $\mathrm{D}_{\mathrm{i}}$ " and the $\mathrm{F}$ value are dependent on the sample size; therefore, the critical value of " $\mathrm{D}_{i}$ " (value where high influence begins to occur) varies.

confidence interval (95-percent) The confidence interval represents a band within which there is a 95-percent probability that estimates of the mean bankfull-channel geometry or discharge for a particular drainage area will occur.

drainage area That area, measured in a horizontal plane, enclosed by a topographic divide from which direct surface runoff from precipitation normally drains by gravity into the stream above the specified point.
Kolmogorov-Smirnov Goodness of Fit Test A statistical test of the underlying regression assumption that residuals (observed value minus predicted value) are normally distributed. The $\mathrm{p}$ value from this test measures the probability that a departure from normality occurs by chance.

natural-stream design For the purposes of this study, naturalstream design is defined as a design intended to restore an impaired stream reach to a state that can transport sediment load and runoff provided to it from upstream without excessive aggradation or degradation while maintaining habitat and aesthetics consistent with that found in an unimpaired reach subjected to similar runoff conditions.

physiographic province A region of which all parts are similar in geologic structure and climate and which has consequently had a unified geomorphic history; a region whose pattern of relief features or landforms differs significantly from that of adjacent regions.

regional curve An ordinary least-squares regression expressed as a power function that relates drainage area to selected bankfull response variables.

regulation A condition where streamflow is controlled by an upstream human-made feature.

residual standard error Measures the dispersion of the data around a regional curve and commonly is stated as the standard deviation of residuals (Helsel and Hirsch, 2002). Residual standard errors are not comparable between relations of different bankfull response variables and drainage area. For example, the residual standard error for the relation between bankfull crosssectional area and drainage area cannot be compared directly to the residual standard error for the relation between bankfull depth and drainage area because the magnitude of bankfull cross-sectional area is much greater than bankfull depth.

stream reach A section of stream usually extending between 10 and 20 bankfull widths in length.

stream restoration Adjusting stream dimensions, pattern, and profile to a condition where it effectively accommodates a range of streamflow and sediment and supports diverse habitat.

watershed For this report, used interchangeably with drainage area. 